\title{
AN IMPROVED TOPONOGOV COMPARISON THEOREM \\ FOR NONNEGATIVELY CURVED MANIFOLDS
}

\author{
DOUG ELERATH
}

\section{INTRODUCTION}

The main result of this paper is a global comparison theorem of Toponogov type which gives improved estimates in open nonnegatively curved manifolds. Previously, estimates on such a manifold could only be made by comparison with $\mathbf{R}^{2}$. It is apparent that further improvements can be made, which lead towards an integral formulation of the theorem.

The application presented in section 5 is intended primarily as an example of the usefulness of this comparison theorem, and not as the optimal approach to the conjecture of Cheeger and Gromoll. It does in fact seem to me that this approach must be stretched to its limit if it is intended to be used in an effort to prove the conjecture.

The following is a list of the contents of this paper:

\section{PRELIMINARIES}

\subsection{Notation}

1.2. Curvature and conjugate points

1.3. Rauch-Berger comparison theorem

2. THE CUT LOCUS ON A FLATTENING SURFACE OF REVOLUTION

2.1. Preliminaries

2.2. Results

2.3. Example

\section{HINGES, TRIANGLES AND BRANCHED COVERINGS}

3.1. Definitions and notation

3.2. Hinges in flattening surfaces

3.3. Hinges in branched coverings of flattening surfaces

Communicated by H. Samelson, July 21, 1978. This represents part of a Ph.D. dissertation written under the guidance of Professor Jeff Cheeger at Stony Brook. It is a pleasure for the author to acknowledge the influence that Professor Cheeger has had on this work. 


\section{GLOBAL COMPARISON THEOREMS}

\subsection{Definitions and notation}

4.2. Two global comparison theorems

\section{MANIFOLDS DIFFEOMORPHIC TO EUCLIDEAN SPACE}

5.1. The soul of a manifold

5.2. Compact half spaces and shriveled souls.

Portions, particularly $\S \S 3$ and 4 , are rather technical; but this is perhaps unavoidable.

\section{PRELIMINARIES}

This section recalls certain elementary aspects of differential geometry which are cited in the body of this paper, and establishes notation to be used throughout. A proof will be given only for Corollary 1.3.2 which, to the best of the author's knowledge, is not standard. All proofs and definitions which are omitted from this section are most easily found in Cheeger and Ebin [2].

\subsection{Notation}

$M^{n}$ will denote a complete $n$-dimensional Riemannian manifold with metric $\langle$,$\rangle and Levi-Civita connection \nabla$. Unless otherwise specified, all geodesics in $M$ will be assumed to be normal; $\left\|\gamma^{\prime}\right\|=1$, where $\gamma^{\prime}(t)$ denotes the tangent vector to $\gamma$ at $\gamma(t)$. If $x$ and $y$ are points in $M$, then $\operatorname{Cur}(x, y)$, $\operatorname{Geo}(x, y)$ and $\mathrm{Geo}_{m}(x, y)$ will denote respectively those curves, geodesics and globally minimal geodesics which begin at $x$ and end at $y$. Elements of $\operatorname{Cur}(x, y)$ are required to be at least piecewise smooth. Thus if $\gamma \in \operatorname{Cur}(x, y)$ is parameterized on $[0, b]$, then $\gamma(0)=x$ and $\gamma(b)=y$. Similarly, if $N \subseteq M$, then $\operatorname{Cur}(N), \operatorname{Geo}(N)$ and $\mathrm{Geo}_{m}(N)$ will denote respectively those curves, geodesics and globally minimal geodesics which have images contained in $N$. Again, elements of $\operatorname{Cur}(N)$ are required to be at least piecewise smooth. If the parameter range of any curve $\gamma$ is not specified, it is understood to be $[0, L[\gamma]]$, where $L[\gamma]$ denotes the length of $\gamma$. Finally, if $\gamma \in \operatorname{Geo}(x, y)$ and $\eta \in \operatorname{Geo}(x, z)$, we let $\Varangle(\gamma, \eta)(x)=\operatorname{arc} \cos \left\langle\gamma^{\prime}(0), \eta^{\prime}(0)\right\rangle, 0 \leqslant \Varangle(\gamma, \eta)<\pi$.

If $\gamma:[0, b] \rightarrow M$, define $-\gamma:[0, b] \rightarrow M$ by $-\gamma(t)=\gamma(b-t)$. If $\gamma_{1} \in$ $\operatorname{Cur}(x, y)$ and $\gamma_{2} \in \operatorname{Cur}(y, z)$ are parameterized on $\left[0, b_{1}\right]$ and $\left[0, b_{2}\right]$ respectively, define $\gamma_{1} \vee \gamma_{2}:\left[0, b_{1}+b_{2}\right] \rightarrow M$ by

$$
\gamma_{1} \vee \gamma_{2}= \begin{cases}\gamma_{1}(t) & \text { if } t \in\left[0, b_{1}\right], \\ \gamma_{2}\left(t-b_{1}\right) & \text { if } t \in\left[b_{1}, b_{1}+b_{2}\right]\end{cases}
$$

Thus $\gamma_{1} \vee \gamma_{2} \in \operatorname{Cur}(x, y)$. 
Sometimes we shall, without further warning, let the same symbol denote both a curve and its image.

If $N \subset M$ is any subset and $r \in \mathbf{R}$ is any positive real number, $T_{r}(N)=\{x$ $\in M \mid d(x, N)<r\}$ is an (open) tubular neighborhood of $N$. Here, of course, $d$ denotes distance in the metric space structure induced by the connection.

\subsection{Curvature and conjugate points}

If $x \in M$, and $\sigma$ is a plane in $M_{x}$, then $K(\sigma)$ will denote the sectional curvature of $M$ at $x$ determined by any two vectors spanning $\sigma$. The notation $K(x)$ will occur only in conjunction with an inequality sign, and will denote either $\inf \{K(\sigma)\}$ or $\sup \{K(\sigma)\}$ over all planes $\sigma$ in $M_{x}$ according as we have $K(x) \geqslant$ or $K(x) \leqslant$.

If $\gamma \in \operatorname{Geo}(x, y)$, we will say that $\gamma$ is free of conjugate points when we mean that $\gamma(t)$ is not conjugate to $x$ along $\gamma$ for any $t$ in the domain of $\gamma$.

1.2.1. Lemma. Let $M^{n}$ and $M_{0}^{n+k}$ be Riemannian manifolds, let $\gamma:[0, b] \rightarrow$ $M$ and $\gamma_{0}:[0, b] \rightarrow M_{0}$ be normal geodesics, and suppose that $K\left(\gamma_{0}(t)\right)>$ $K(\gamma(t))$ for all $t \in[0, b]$. Then if $\gamma_{0}$ is free of conjugate points, so is $\gamma$.

1.2.2. Remark. If $M$ and $M_{0}$ are both 2-dimensional this lemma follows immediately from the Sturm comparison theorem for second order ordinary differential equations. See, for example, [10, p. 118]. Thus, despite the application of this relatively modern result [ca. 1950] to surfaces in $\$ 2$, the techniques there should be considered entirely classical.

\subsection{Rauch-Berger comparison theorem}

Suppose that $x$ and $y$ are points in $M^{n}$. If $\gamma \in \operatorname{Geo}(x, y)$, we will say that $\gamma$ is free of focal points when we mean that $\gamma(t)$ is not a focal point of the $(n-1)$-dimensional embedded submanifold defined by restricting exp to a sufficiently small neighborhood of $0 \in \gamma^{\prime}(0)^{\perp} \subset M_{x}$.

1.3.1. Theorem (Berger). Let $M^{n}$ and $M_{0}^{n+k}$ be Riemannian manifolds. Let $\gamma:[0, b] \rightarrow M$ and $\gamma_{0}:[0, b] \rightarrow M_{0}$ be normal geodesics with $\gamma_{0}$ free of focal points. Assume for each $t \in[0, b]$, each $v \in M_{\gamma(t)}$ and each $v_{0} \in M_{0_{\gamma}(t)}$ that the sectional curvatures of the sections $\sigma$ and $\sigma_{0}$ spanned respectively by $\left(\gamma^{\prime}(t), v\right)$ and $\left(\gamma_{0}^{\prime}(t), v_{0}\right)$ satisfy $K\left(\sigma_{0}\right) \geqslant K(\sigma)$. Let $T(t)=\gamma^{\prime}(t)$ and $T_{0}(t)=\gamma_{0}^{\prime}(t)$, and let $J$ and $J_{0}$ be Jacobi fields along $\gamma$ and $\gamma_{0}$ respectively satisfying

(1) $\left(\nabla_{T} J\right)(0)$ and $\left(\nabla_{T_{0}} J_{0}\right)(0)$ are tangent to $\gamma$ and $\gamma_{0}$ respectively,

(2) $\left\|\nabla_{T} J\right\|(0)=\left\|\nabla_{T_{0}} J_{0}\right\|(0)$,

(3) $\langle T, J\rangle(0)=\left\langle T_{0}, J_{0}\right\rangle(0)$, and

(4) $\|J\|(0)=\left\|J_{0}\right\|(0)$.

Then $\|J\|(t)>\left\|J_{0}\right\|(t)$ for each $t \in[0, b]$, 
The following corollary, although well known, appears nowhere in the literature, so a proof of it is given here.

1.3.2. Corollary. Let $\gamma:[0, b] \rightarrow M^{n}$ and $\gamma_{0}:[0, b] \rightarrow M_{0}^{n+k}$ be normal geodesics, and let $E$ and $E_{0}$ be parallel unit vector fields along $\gamma$ and $\gamma_{0}$ respecively with $\left\langle E, \gamma^{\prime}\right\rangle=\left\langle E_{0}, \gamma_{0}^{\prime}\right\rangle$. Suppose that $c:[0, b] \rightarrow M$ and $c_{0}:[0, b]$ $\rightarrow M_{0}$ are defined by

$$
\begin{aligned}
c(t) & =\exp _{\gamma(t)}(f(t) E(t)), \\
c_{0}(t) & =\exp _{\gamma_{0}(t)}\left(f(t) E_{0}(t)\right),
\end{aligned}
$$

where $f:[0, b] \rightarrow \mathbf{R}$ is smooth. Let $\eta_{t}:[0,1] \rightarrow M$ and $\eta_{0 t}:[0,1] \rightarrow M_{0}$ be defined by

$$
\begin{aligned}
\eta_{t}(s) & =\exp _{\gamma(t)}(s f(t) E(t)), \\
\eta_{0 t}(s) & =\exp _{\gamma_{0}(t)}\left(s f(t) E_{0}(t)\right) .
\end{aligned}
$$

Assume that for each $(t, s) \in[0, b] \times[0,1], K\left(\eta_{t}(s)\right) \leqslant K\left(\eta_{0 t}(s)\right)$, and that for each $t \in[0, b], \eta_{0 t}$ is free of focal points. Then $L[c]>L\left[c_{0}\right]$.

Proof. Since $c$ and $c_{0}$ are both parameterized on $[0, b]$, it suffices to compare the lengths of their tangent vectors.

If $t_{1} \in[0, b]$ is fixed, and $\alpha:(-\varepsilon, \varepsilon) \times[0,1] \rightarrow M$ is a variation given by $\gamma(t, s)+\eta_{t_{1}+t}(s)$, then $V(s)=(\partial \alpha / \partial t)(0, s)$ is a Jacobi field along $\eta_{t_{1}}$ with end values $V(0)=\gamma^{\prime}\left(t_{1}\right)$ and $V(1)=c^{\prime}\left(t_{1}\right)$. Note further that since $\nabla_{\gamma^{\prime}} E=0$ and $\eta_{t}^{\prime}(0)=f(t) E(t)$,

$$
\begin{aligned}
\left(\nabla_{\eta_{t i}} V\right)(0) & =\left[\nabla_{V(0)} f(t) E(t)\right]\left(t_{1}\right) \\
& =\left[V(f(t)) E(t)+f(t) \nabla_{\gamma^{\prime}} E(t)\right]\left(t_{1}\right) \\
& =f^{\prime}\left(t_{1}\right) E\left(t_{1}\right) .
\end{aligned}
$$

Similarly, we find that $\left(\nabla_{\eta_{0}^{\prime t_{1}}} V_{0}\right)(0)=f^{\prime}\left(t_{1}\right) E_{0}\left(t_{1}\right)$. This shows that $V$ and $V_{0}$ satisfy condition (1) of Theorem 1.3.1. Evidently they satisfy the other conditions, and thus $\|V\|(1) \geqslant\left\|V_{0}\right\|(1)$. Hence $\left\|c^{\prime}\left(t_{1}\right)\right\| \geqslant\left\|c_{0}^{\prime}\left(t_{1}\right)\right\|$ for any $t_{1} \in[0, b]$, and thus $L[c] \geqslant L\left[c_{0}\right]$.

Remark. Note that $\left\langle E, \gamma^{\prime}\right\rangle$ and $\left\langle E_{0}, \gamma_{0}^{\prime}\right\rangle$ were not required to be zero in this corollary.

\section{THE CUT LOCUS ON A FLATTENING SURFACE OF REVOLUTION}

Interest in the cut locus on surfaces dates back to Poincaré [15], and Myers [13], [14]. They both proved that the cut locus of a point $x$ on a surface $S$ can contain no closed curve, and that the end points of the cut locus of $x$ are 
conjugate to $x$. In addition, Myers proved that on an analytic surface the cut locus of any point is a tree with finitely many nodes in any compact subset of $S$.

To illustrate the notion of the cut locus, Myers describes the cut locus on several standard surfaces such as the plane, the sphere and the ellipsoid, and then states that "examples of well-known simply connected surfaces on which the (cut) locus assumes a complicated, but determinable, form are naturally hard to give." It is in fact extremely difficult to calculate the cut locus on a specific surface unless the surface is quite nice. Gluck and Singer [8] have recently shown that any smooth manifold of dimension $\geqslant 2$ can be given a Riemannian metric with a non-triangulable cut locus, and they construct such a metric on the 2-sphere.

Previously, "quite nice" has commonly meant a quadric surface, or perhaps a cylinder or torus. Even in the case of a surface of revolution, where geodesics can be explicitly exhibited in integral form, the cut locus is not generally known. This problem was, however, solved for paraboloids and hyperboloids in a beautiful paper by von Mangoldt [12]. In this paper he sidesteps the task of calculating the elliptic integrals defining geodesics on these surfaces, and computes the conjugate locus directly. The cut locus is then immediately apparent.

In this section we will extend the results of von Mangoldt to the class of flattening surfaces of revolution. The techniques will not be his; but, as mentioned in $\S 1$, the techniques are essentially classical.

\subsection{Preliminaries}

Let $(r, \theta, z): \mathbf{R}^{3} \rightarrow[0, \infty) \times[0,2 \pi) \times \mathbf{R}$ be cylindrical coordinates. Let $S^{2} \subset \mathbf{R}^{3}$ be a smooth surface of revolution about the $z$-axis, with the induced metric, such that $\left.(r, \theta)\right|_{S}$ is $1-1$. Let $p=\left.r\right|_{S} ^{-1}(0)$ be the vertex of $S$, and let $\rho: S \rightarrow \mathbf{R}$ be defined by $\rho(s)=d(p, s)$, where the distance $d$ is measured in the surface $S$.

Using (a) the integral expression for a geodesic on a surface of revolution and (b) Clairaut's theorem (see for example [7]), one can easily deduce the following qualitative description of the behavior of geodesics on $S$.

2.1.1. Description. On each infinite normal geodesic $\gamma: \mathbf{R} \rightarrow S$ there is a unique point, denoted $\sigma_{\gamma}$, which is nearest to the vertex of $S$, about which $\gamma$ is symmetric, and at which $\gamma$ is tangent to a parallel of $S$. The two branches of $\gamma$ 
proceed in either direction from this point and spiral in opposite senses around the axis of $S$ heading monotonically towards infinity, so that $\rho \circ \gamma$ satisfies a maximum principle.

Ling [11] provides a more detailed discussion of geodesics on a slightly more restricted class of surfaces.

2.1.2. Definition. Such a surface of revolution $S$ will be said to be flattening if $d K / d \rho \leqslant 0$, where $K$ denotes the Gauss curvature of the surface and $\rho$ was defined above.

Thus we add to our notion of a surface of revolution the requirement that the curvature decreases as distance from the vertex increases.

2.1.3. Notation. We let $S$ be a flattening surface of revolution, and set

(1) $S_{+}=S \cap \theta^{-1}(0, \pi)$,

(2) $P_{x}=$ the parallel ( $z=$ constant) through a point $x \in S$,

(3) $\mu_{x}=$ the meridian $\left(\theta=\right.$ constant) through a point $x \in S$ with $\mu_{x}(0)=$ $p$,

(4) $\hat{\mu}_{x}=$ the meridian opposite $x$, i.e., $\hat{\mu}_{x}(t)=\exp _{p}\left(-t \mu_{x}^{\prime}(0)\right)$. If $\gamma \in$ $\operatorname{Geo}(S)$, domain $(\gamma)=\mathbf{R}$, then

(5) $\tau_{\gamma}=\inf r(\gamma(t)), t \in \mathbf{R}$,

(6) $\sigma_{\gamma}=$ that unique point in $S$ on $\gamma$ for which $r\left(\sigma_{\gamma}\right)=\tau_{\gamma}$. If $\gamma \in \operatorname{Geo}(S)$, domain $(\gamma) \neq \mathbf{R}$, then

(7) $\bar{\gamma} \in \operatorname{Geo}(S)$ denotes the unique extension of $\gamma$ so that $\operatorname{Im}(\gamma) \subset \operatorname{Im}(\bar{\gamma})$ and domain $(\bar{\gamma})=\mathbf{R}$,

(5') $\tau_{\gamma}=\tau_{\bar{\gamma}}$,

(6) $\sigma_{\gamma}=\sigma_{\bar{\gamma}}$.

Suppose that $\gamma \in \operatorname{Geo}\left(S_{+}\right)$, domain $(\gamma)=[0, b], \gamma$ not a meridian.

(8) $\theta_{\gamma}:[0, b] \rightarrow[0, \pi)$ by $\theta_{\gamma}(t)=|\theta(\gamma(0))|$. That $\theta_{\gamma}$ is $1-1$ follows from Description 2.1.1.

(9) $F_{\gamma}: \operatorname{Im}\left(\theta_{\gamma}\right) \rightarrow \rho(\gamma)$ by $F_{\gamma}=\rho \circ \gamma \circ \theta_{\gamma}^{-1}$, so that $\gamma$ can be viewed as the graph of $F_{\gamma}$.

2.1.4. Definition. If $\gamma, \eta \in \operatorname{Geo}\left(S_{+}\right.$), we say that $\eta$ lies below (above) $\gamma$ if (i) $\theta(\gamma) \subset \theta(\eta)$, and (ii) for each $t \in$ domain $(\gamma), \rho\left(\eta \cap \mu_{\gamma(t)}\right)<(>) \rho(\gamma(t))$. The same terminology will be used if $\gamma$ is simply a point. Note that if $\eta$ lies below $\gamma$, it is not necessarily the case that $\gamma$ lies above $\eta$.

Finally, we need the following result which is implicit in the paper [14] of Myers.

2.1.5. Lemma (Myers). Suppose $M^{2}$ is a smooth complete surface, and $x \in M, y \in M$. Given $g \in \mathrm{Geo}_{m}(x, y), h \in \mathrm{Geo}_{m}(x, y)$ with $g \cup h$ the boundary of a simply connected region $D$, then there exists $z \in C l(D)$ such that $x$ and $z$ are conjugate along $a$ geodesic $g_{0} \in \mathrm{Geo}_{m}(C l(D))$. 


\subsection{Results}

In Lemmas 2.2.2, 2.2.3 and 2.2.4 we assume the following data.

2.2.1. Data (see Fig. 1).

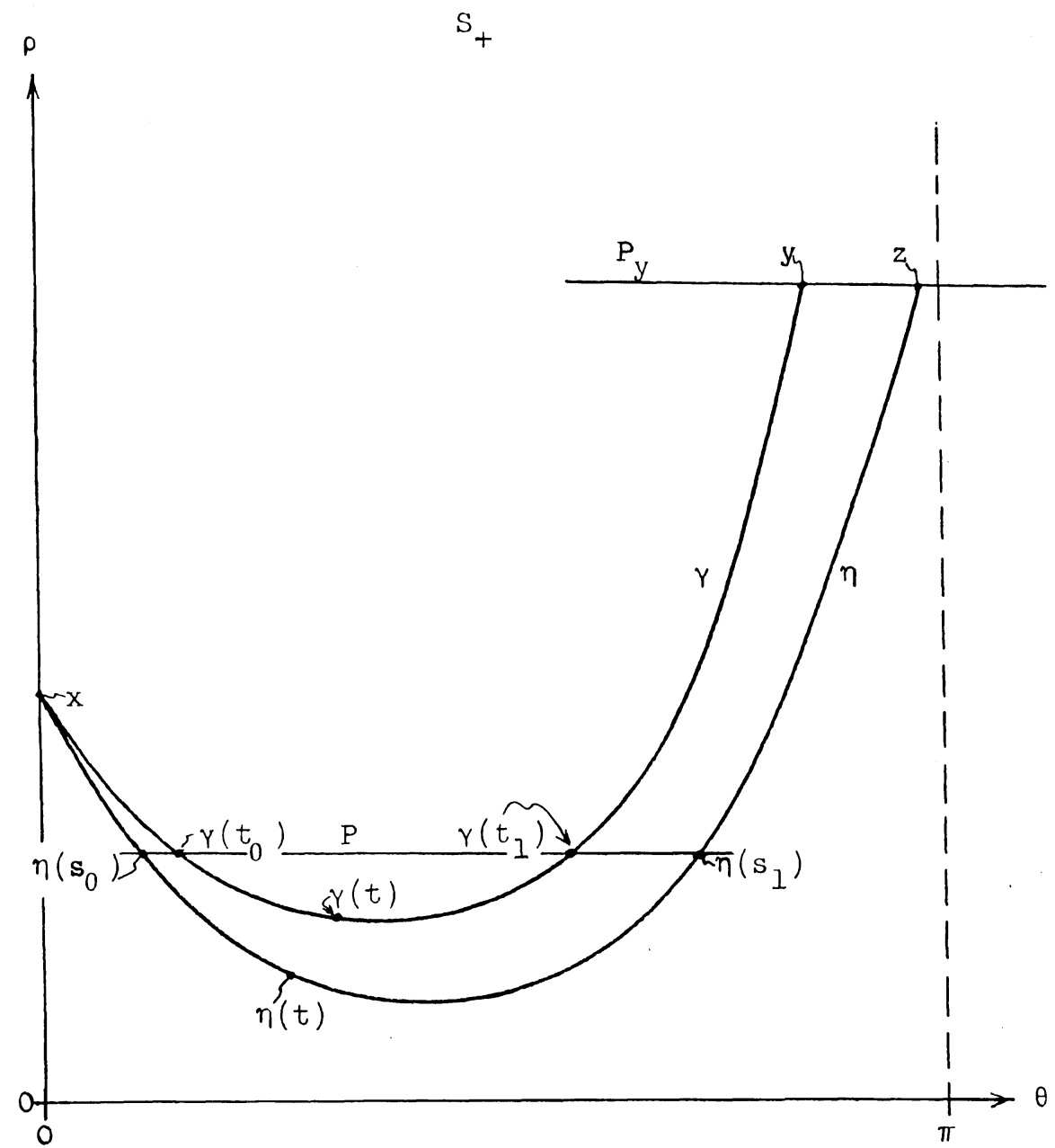

FIG. 1

(1) $x \in \partial S_{+}$with $\theta(x)=0$;

(2) $y \in S_{+}$with $\rho(x) \leqslant \rho(y)$;

(3) $\gamma \in \mathrm{Geo}_{m}(x, y), \gamma:[0, a] \rightarrow S$;

(4) $z \in P_{y} \cap S_{+}$is such that $\theta(z)>\theta(y)\left(S_{+}\right.$is open);

(5) $\eta \in \mathrm{Geo}_{m}(x, z), \eta:[0, b] \rightarrow S$.

The following three technical lemmas explore in detail the behavior of the geodesics $\gamma$ and $\eta$. 
2.2.2. Lemma. $\quad \gamma, \eta \subset S_{+}$and $\eta$ lies below $\gamma$.

Proof. The first claim follows immediately upon observing that (i) $S$ is symmetric with respect to reflection through the plane determined by $\partial S_{+}$, and (ii) $\gamma$ and $\eta$ are minimal. Thus if, for example, $h$ is any geodesic from $x$ to $z$, we may reflect all portions of $h$ not in $S_{+}$through the plane determined by $\partial S_{+}$. If $h \not \subset C l\left(S_{+}\right)$, the result is a non-smooth curve from $x$ to $z$ of the same length as $h$, and hence $h$ could not have been minimal. (This shows that $S_{+}$is convex.)

Since $\theta(z)>\theta(y), \gamma, \eta \subset S_{+}$and $\theta_{\gamma}, \theta_{\eta}$ are $1-1$, we have $\theta(\gamma) \subset \theta(\eta)$. Since both $\gamma$ and $\eta$ are minimal, $\eta \cap \gamma=\{x\}$. Thus $\eta$ lies either above $\gamma$ or below $\gamma$. Let $H(t)=F_{\eta}\left(t+\theta_{\eta}(0)\right)$ and $G(t)=F_{\gamma}\left(t+\theta_{\gamma}(0)\right)$ map $\theta(\gamma)$ to R. If $\eta$ lies above $\gamma, H>G$, and in particular $H(\theta(y))>G(\theta(y))$. Then $H(0)<H(\theta(z))$ $<H(\theta(y))$, while $0<\theta(y)<\theta(z)$. This is of course impossible since $H$ cannot achieve a maximum at an interior point (cf. Description 2.1.1).

2.2.3. Lemma. $L[\gamma]<L[\eta]$.

Proof. Suppose that $P_{y}$ is parameterized, not by arc length, but such that $\theta(z)-\theta\left(P_{y}(s)\right)=s$, and let $z_{s}=P_{y}(s)$. From $\eta^{\prime}(\theta)>0, P_{y}^{\prime}(\theta)<0$ and $P_{y}^{\prime}(\rho)$ $=0$, it follows that $\left\langle\eta^{\prime}, P_{y}^{\prime}(z)\right\rangle<0$. Now choose a smooth variation $\alpha:(-\delta, \delta) \times[0, b] \rightarrow S$ of $\eta$ with $\alpha(s, 0)=x, \alpha(s, b)=z_{s}$ and $\alpha(0, t)=\eta(t)$. Then $\left.\left\langle\partial / \partial s(\alpha), \eta^{\prime}\right\rangle\right|_{s=0 ; t=b}=\left\langle P_{y}^{\prime}, \eta^{\prime}\right\rangle(z)<0$, and so the first variation formula implies that $\partial /\left.\partial s\right|_{s=0} L[\alpha,(s, \cdot)]<0$.

Since both Lemma 2.2.2 and the argument of the preceding paragraph apply as well to each $\eta_{s} \in \operatorname{Geo}_{m}\left(x, z_{s}\right)$ for all relevant $s$, i.e., when $\theta(y)<z_{s}$ $<\theta(z)$, it follows that $L\left[\eta_{s}\right]$ decreases monotonically as $s$ increases. Thus $L[\gamma]<L[\eta]$.

2.2.4. Lemma. $\rho(\gamma(t))>\rho(\eta(t))$ for each $t>0$ in the domain of $\gamma$.

Proof. Let $P$ be any parallel with $\tau_{\gamma}<\rho(P) \leqslant \rho(y)$. Let $t_{1}$ be such that $\gamma\left(t_{1}\right) \in P$ and $F_{\gamma}^{\prime}\left(t_{1}\right)>0$, and thus for all $t>t_{1}, \rho(\gamma(t))>\rho(P)$. (That such a $t_{1}$ exists is clear from Data 2.2.1 and Description 2.1.1). Let $s_{1}$ be similarly defined in terms of $\eta$. The argument given in Lemma 2.2.3 applies, mutatis mutandis, to show that $t_{1}<s_{1}$.

If $P \cap \gamma$ is but a single point, $P \cap \eta$ will likewise be a single point, and necessarily these intersections will be the $\gamma\left(t_{1}\right)$ and $\eta\left(s_{1}\right)$ described in the previous paragraph $(\rho(y) p(z)=\rho(x))$. Thus, since $P$ lies above $\eta \mid\left[0, s_{1}\right]$, $\rho\left(\eta\left(t_{1}\right)\right)<\rho\left(\eta\left(s_{1}\right)\right)=\rho\left(\gamma\left(t_{1}\right)\right)$.

If $P \cap \gamma$ is two points (the only other case), $P \cap \eta$ will also be two points, and it must be that $F_{\gamma}^{\prime}(0)<0$. Define $t_{0}$ so that $\gamma\left(t_{0}\right) \in P$ and $F_{\gamma}^{\prime}\left(t_{0}\right)<0$, and let $s_{0}$ be similarly defined in terms of $\eta$. Thus if $t_{0}<t<t_{1}, \rho(\gamma(t))<\rho(P)$; while if $s_{0}<t<s_{1}, \rho(\eta(t))<\rho(P)$. Let $w=\gamma\left(t_{0}\right)$ and let $P_{w}$ be parameterized so that $\theta(w)-\theta\left(P_{w}(s)\right)=s$. Let $w_{s}=P_{w}(s)$ and let $\gamma_{s} \in$ 
$\mathrm{Geo}_{m}\left(x, w_{s}\right)$ for $0 \leqslant s<\theta(w)$. It is easily seen that $F_{\gamma_{s}}^{\prime}\left(\theta\left(w_{s}\right)\right)<0$ : Rotate $\gamma$ so that $\gamma\left(t_{0}\right)$ passes through $w_{s}$; it is then clear that $\gamma_{s}$ is trapped above this rotated version of $\gamma$, and thus $F_{\gamma_{s}}^{\prime}\left(\theta\left(w_{s}\right)\right)<F_{\gamma}^{\prime}\left(t_{0}\right)<0$. Thus $\left\langle\gamma_{s}^{\prime}, P^{\prime}\right\rangle_{w_{s}}<0$, and using a first variation argument as in Lemma 2.2.3 we see that $s_{0}<t_{0}$ (the geodesics are parameterized by arc length).

Now given any $t \in \operatorname{domain}(\gamma)$ we construct $P_{\gamma(t)}$, with $s_{0}, s_{1}, t_{0}$ and $t_{1}$ as above. The foregoing argument implies that $s_{0}<t_{0} \leqslant t \leqslant t_{1}<s_{1}$, and thus that $\rho(\eta(t))<\rho\left(\eta\left(s_{1}\right)\right)=\rho\left(\gamma\left(t_{1}\right)\right)=\rho(\gamma(t))$. q.e.d.

In the following we let $C(x)$ denote the cut locus of $x$.

2.2.5. Theorem. Let $S$ be a flattening surface of revolution and $x \in S$. Then $C(x) \subset \hat{\mu}_{x}$.

Proof. Without loss of generality we may suppose that $x \in \partial S_{+}, \theta\left(S_{+}\right)=$ $(0, \pi)$, and $\theta(x)=0$. If $C(x) \cap S_{+} \neq \varnothing$, then the convexity of $S_{+}$(shown in Lemma 2.2.2), the characterization of cut points given in Lemma 5.2 of [2], and Lemma 2.1.5 together imply that there exists a $y \in S_{+}$such that $x$ and $y$ are conjugate along a minimal geodesic in $S_{+}$. By relabeling, if necessary, we may suppose that $\rho(x)<\rho(y)$. We will now see that such a situation, i.e., $x$ conjugate to such a $y$ in $S_{+}$, is not possible, and thus that $C(x) \cap S_{+}=\varnothing$. The theorem is then apparent.

So, suppose we are given Data 2.2.1 on $S$. Then, since $S$ is flattening, $\rho(\eta(t))<\rho(\gamma(t))$ for $t \in \operatorname{domain}(\gamma)$ implies that $K(\eta(t))>K(\gamma(t))$. Moreover, since $\eta$ is minimal, $x$ can have no conjugate points along $\eta$ for a distance equal to $L[\eta]>L[\gamma]$. Thus Lemma 1.2.1 shows that $y$ is not conjugate to $x$ along $\gamma$, and we are done.

\subsection{Example}

This example shows that Theorem 2.2.5 is sharp in the following sense: Given $\delta>0$, there exist a surface of revolution $S$ and a point $x \in \partial S_{+}$such that (i) $d K / d \rho<0$ except on a set $E \subset S$, (ii) $m(E)<\delta$, where $m$ denotes Lebesgue measure, and (iii) $C(x) \cap S_{+} \neq \varnothing$. Equivalently, by multiplying the metric by a constant, we can replace (ii) by (ii') $d K /\left.d \rho\right|_{E}<\delta$.

We will construct a surface which is only piecewise smooth, and in fact does not satisfy our requirements for a surface of revolution (cf. §2.1.). But standard approximation theorems, see for example Aleksandrov and Zalgaller [1], imply that this is sufficient.

2.3.1. Let $f:[0, \infty) \rightarrow \mathbf{R}^{2}$ be the curve

$$
f(t)= \begin{cases}(t, 0) & 0 \leqslant t \leqslant 1, \\ (1, t-1) & 1 \leqslant t,\end{cases}
$$


and let $S$ be the surface generated by revolving $f$ about the vertical axis. See Fig. 2. Let $\gamma: \mathbf{R} \rightarrow S$ be a geodesic with $0<\tau_{\gamma}<1$, and $\sigma_{\gamma}=\left(\tau_{\gamma}, 0,0\right)$. Then for some $\varepsilon>0, \gamma \mid\left[-t_{0}, t_{0}\right] \subset S_{+}$, where $t_{0}=\left(1-\tau_{\gamma}^{2}\right)^{1 / 2}+\varepsilon$, and both $\gamma\left(t_{0}\right)$ and $\gamma\left(-t_{0}\right)$ are in the cylindrical part of $S$. Thus there is another geodesic $\eta \in \operatorname{Geo}\left(\gamma\left(-t_{0}\right), \gamma\left(t_{0}\right)\right) \cap \operatorname{Geo}\left(S_{+}\right)$which is distinct from $\gamma$, and so $C\left(\gamma\left(t_{0}\right)\right) \cap$ $S_{+} \neq \varnothing$. Now we can find an approximation to $S$ for which (i) and (ii), or (i) and (ii') hold, and a point $x$ on the approximation such that (iii) holds.

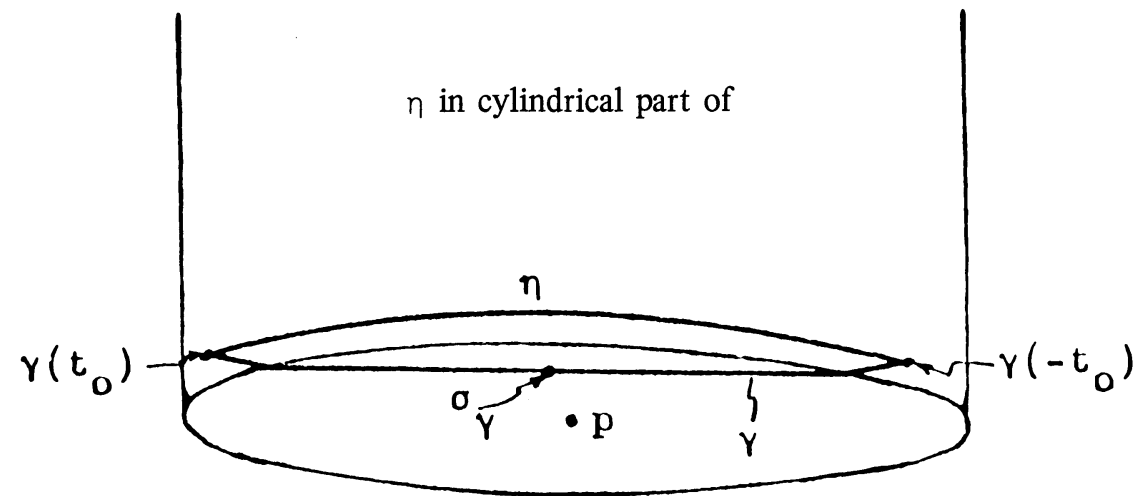

FIG. 2

2.3.2. With a little more care, (i), (ii) and (ii') can all be made to hold simultaneously. Here we let $f_{\theta}:[0, \infty) \rightarrow \mathbf{R}^{2}$ be the curve

$$
f_{\theta}(t)= \begin{cases}(1-\cos (t), \sin (t)) & 0 \leqslant t \leqslant \theta \leqslant \pi / 2 \\ (1-\cos (\theta), t-\theta+\sin (\theta)) & \theta \leqslant t\end{cases}
$$

and let $S_{\theta}$ be the surface generated by revolving $f$ about the vertical axis. Just as in $\$ 2.3 .1$ we find, for a given $\theta<\pi / 2$, that there is an $x \in \partial S_{+}$with $C(x) \cap S_{+} \neq \varnothing$.

If we now approximate $S_{\theta}$ by a smooth surface of revolution $T_{\theta}$ which satisfies (i) and (ii') ( $T_{\theta}$ may differ from $S_{\theta}$ by a conformal factor), then, if $\theta$ is sufficiently near $\pi / 2, T_{\theta}$ will satisfy (i), (ii), (ii') and (iii).

\section{HINGES, TRIANGLES AND BRANCHED COVERINGS}

In this section we define hinges and geodesic triangles in a Riemannian manifold, and then prove several technical lemmas which examine hinges more closely on flattening surfaces and their branched coverings. The idea is to replace part (1) of the proof of Toponogov's theorem as presented in [2] with a lemma appropriate to a flattening surface of revolution. The reasons for going to the branched coverings are somewhat subtle, and will be indicated later in the paper. 


\subsection{Definitions and notation}

Throughout this section, any geodesic written $\gamma_{i}$ is assumed to be parameterized on $\left[0, b_{i}\right]$, and we let $e_{i}=\gamma_{i}\left(b_{i}\right)$. Furthermore, if $\left\{\gamma_{i}^{\alpha}\right\}$ is a family of geodesics which is parameterized by $\alpha$ with each $\gamma_{i}^{\alpha}$ parameterized on $\left[0, b_{i}\right]$, then set $e_{i}^{\alpha}=\gamma_{i}^{\alpha}\left(b_{i}\right)$.

If $(M, d)$ is any metric space with $A \subset M, B \subset M$, let $d_{A}(B)=$ $\sup \{d(A, x) \mid x \in B\}$, and define the Hausdorff distance between $A$ and $B$ by

$$
\operatorname{hd}(A, B)=\max \left\{d_{A}(B), d_{B}(A)\right\} \text {. }
$$

If $S$ is a surface of revolution and $\gamma \in \operatorname{Geo}(S)$, we may extend 2.1.3(8) and define $\theta_{\gamma}$ as follows: We may suppose that $0 \in \operatorname{domain}(\gamma)$. If $x \in \operatorname{range}(\gamma)$, we may orient $S$ in $\mathbf{R}^{3}$ so that $x \in \partial S_{+}, \theta(x)=0$. Let $(a, b)$ be the domain of the connected component of $\left.\gamma\right|_{s_{+}}$such that $\gamma(a)=x$. Then we require that (i) $\theta_{\gamma}(0)=0$ and (ii) if $t \in(a, b), \theta_{\gamma}(t)-\theta_{\gamma}(a)=\theta(t)$. It is easily seen that $\theta_{\gamma}$ is 1-1 for any nonmeridional geodesic $\gamma$ on $S$.

Let $M$ be a Riemannian manifold.

3.1.1. Definition. A hinge in $M$ is a triple $\left(\gamma_{1}, \gamma_{2}, \alpha\right)$ with

(1) $\gamma_{i} \in \operatorname{Geo}(M)$,

(2) $e_{0}=\gamma_{1}(0)=\gamma_{2}(0)$, and

(3) $\Varangle\left(\gamma_{1}, \gamma_{2}\right)\left(e_{0}\right)=\alpha$.

By a family of hinges $\left\{\left(\gamma_{1}^{\alpha}, \gamma_{2}, \alpha\right)\right\}$ we mean that for each $\alpha$ in some interval $\left(\gamma_{1}^{\alpha}, \gamma_{2}, \alpha\right)$ is a hinge in $M$ with each $\gamma_{1}^{\alpha}$ parameterized on $\left[0, b_{1}\right]$. Note that $e_{1}^{\alpha}=\gamma_{1}^{\alpha}\left(b_{1}\right)$ is a continuous function of $\alpha$.

Sometimes, given a hinge $\left(\gamma_{1}, \gamma_{2}, \alpha_{0}\right)$, we will speak of increasing or decreasing $\alpha_{0}$ by moving, say, $\gamma_{1}$. This procedure may be viewed formally by constructing the family of hinges $\left\{\left(\gamma_{1}^{\alpha}, \gamma_{2}, \alpha\right)\right\}, \alpha \in[a, b]$, with $\alpha_{0} \in[a, b]$. Then, if $\alpha_{1} \in\left[a, \alpha_{0}\right)$, to "decrease $\alpha_{0}$ to $\alpha_{1}$ " means to consider $\gamma_{1}^{\alpha_{1}}$ to be the geodesic resulting from this "movement".

3.1.2. Definition. A triple of geodesics $\left(\gamma_{0}, \gamma_{1}, \gamma_{2}\right)$ on $M$ is a geodesic triangle (on $M)$ if $\gamma_{i}\left(b_{i}\right)=\gamma_{i+1}(0), i \in \mathbf{Z}_{3}$. Note that if each $\gamma_{i}$ is minimal, then it is apparent that the $\gamma_{i}$ satisfy the triangle inequality, i.e.,

$$
L\left[\gamma_{i}\right]+L\left[\gamma_{i+1}\right] \geqslant L\left[\gamma_{i+2}\right], \quad i \in \mathbf{Z}_{3} \text {. }
$$

\subsection{Hinges in flattening surfaces}

For the remainder of $\S 3, S$ will denote a flattening surface of revolution with vertex $p, R$ will be a fixed positive real number, and attention will be restricted to the compact ball $C l\left(T_{2 R}(p)\right)$ in $S$.

The following lemma is a bit unwieldy, but not without purpose. In this lemma we define a number of constants $\delta_{1}, \cdots, \delta_{5}$ associated to a given 
flattening surface of revolution. Having five instead of just one allows us to more easily point out exactly what hypotheses are needed in later propositions.

3.2.1. Lemma. Let $S$ be a flattening surface of revolution and fix $R \in \mathbf{R}$.

(1) There exists $\delta_{1}>0$ so that if $\gamma \in \operatorname{Geo}\left(T_{2 R}(p)\right)$ with $L([\gamma]<$ $d(\gamma(0), p)+2 \delta_{1}$, then $\gamma$ is free of conjugate points.

(2) There exists $\delta_{2}>0$ so that if $\gamma \in \operatorname{Geo}\left(T_{2 R}(p)\right)$ with $L[\gamma]<\delta_{2}$, then $\gamma$ is free of focal points.

Let $G$ denote the set of normal geodesics in $T_{R}(p)$ such that if $\gamma \in G$, then $L[\gamma]<d(\gamma(0), p)+\delta_{1}$. Each $\gamma \in G$ is parameterized on $[0, L[\gamma]]$.

(3) There exists $\delta_{3}>0$ such that if $\gamma \in G$ and $\eta$ is any geodesic with $\operatorname{hd}(\gamma, \eta)>\delta_{3}$, then $\eta$ is unique between its end points among all those geodesics whose hd distance from $\gamma$ is less than $\delta_{3}$.

(4) There exists $\delta_{4}>0$ such that if $\gamma \in G$ and $z \in T_{\delta_{4}}(\gamma(0))$, then there exists $\eta \in \operatorname{Geo}(\gamma(L[\gamma]), z)$ with $\mathrm{hd}(\eta, \gamma)<\delta_{3}$ and $L[\eta]<L[\gamma]+\delta_{1}$. We may furthermore require that $\Varangle(\gamma, \eta)(\eta(0))<\pi$ unless $\gamma \vee \eta \in \operatorname{Geo}(\gamma(0)$, z) (this is used only in Lemma 3.2.3).

(5) Given $\varepsilon>0$ and $\varphi \in(0, \pi / 2]$, there exists $a \delta_{5} \in\left(0, \delta_{4}\right)$ such that the following holds: Let $\left(\gamma_{1}, \gamma_{2}, \alpha\right)$ be a hinge with $\gamma_{1} \in G, L\left[\gamma_{2}\right]<\delta_{5}$ and $\alpha \in$ $[\varphi, \pi-\varphi]$. Let $\eta$ be the unique geodesic from $e_{1}$ to $e_{2}$ whose hd distance from $\gamma_{1}$ is less than $\delta_{3}$. Recall that $e_{i}$ was defined in $\$ 3.1$. Then, up to reparameterization, $\eta(t)=\exp _{\gamma_{1}}(t) f(t) E(t)$, where $E$ is the parallel unit vector field arrived at by parallel translating $\gamma_{2}^{\prime}$ along $\gamma_{1}$, and $f:[0, b] \rightarrow[0, \varepsilon)$ is smooth.

Proof. In each case we shall produce the $\delta$ for an arbitrary geodesic. Then a uniform $\delta$ can be found using continuity and compactness arguments.

(1) Suppose $\gamma \in \operatorname{Geo}(x, y)$, and let $\mu$ be the meridian segment from $x$ to $p$. Since $x$ and $p$ are not conjugate along $\mu, \mu$ can be extended a small amount beyond $p$ and still be free of conjugate points $(C(x)$ is closed). Thus, assuming tht $\mu$ has been so extended, $L[\mu]>d(x, p)$ and $\mu$ contains the meridian segment from $x$ to $p$. If $p \in \gamma$, we are evidently done. Otherwise, since $\mu$ is minimal from $x$ to $p, d(\mu(t), p)<d(\gamma(t), p)$ for $0<t<d(x, p)$, and thus $d(\mu(t), p)<d(\gamma(t), p)$ holds for $0<t<d(x, p)+2 \delta_{1}$ for some $\delta_{1}>0$. Since $S$ is flattening $K(\mu(t))>K(\gamma(t))$, and the proof is completed by applying Lemma 1.2.1.

(2) This is clearly true on any compact manifold.

(3)-(5) These all follow easily from the facts that $\gamma$ is free of conjugate points, and that $\exp _{x} v$ is a continuous function of both $x \in S$ and $v \in S_{x}$.

Remark. Any $\delta_{i}$ appearing in the remainder of this paper will be assumed to have been chosen in accordance with the above lemma. 
3.2.2. Lemma. Let $\left\{\left(\gamma_{1}^{\alpha}, \gamma_{2}, \alpha\right)\right\}, \alpha \in[a, b]$, be a family of hinges in $S_{+}$. Then $d / d \alpha\left(d\left(e_{1}^{\alpha}, e_{2}\right)\right)>0$ for all $\alpha \in[a, b]$.

Proof. This is a straightforward analogue to step (1) in the proof of Theorem 2.2 in Cheeger and Ebin [2]. Here it is important to observe that if $x \in C l\left(S_{+}\right), C(x) \cap S_{+}=\varnothing$.

3.2.3. Lemma. Let $\left\{\left(\gamma_{1}^{\alpha}, \gamma_{2}, \alpha\right)\right\}, \alpha \in[0, \pi]$, be a family of hinges in $T_{R}(p)$ $\subset$ S. Suppose that

(1) $L\left[\gamma_{1}^{\alpha}\right]<d\left(e_{0}, p\right)+\delta_{1}$, and

(2) $L\left[\gamma_{2}\right]<\delta_{4}$

Let $\gamma^{\alpha}$ denote the unique geodesic from $e_{2}$ to $e_{1}^{\alpha}$ whose hd distance from $\gamma_{1}^{\alpha}$ is less than $\delta_{3}$. Then $(d / d \alpha) L\left[\gamma^{\alpha}\right]>0$ for all $\alpha \in[0, \pi]$.

Proof. Since $\gamma_{1}^{\alpha}$ is free of conjugate points for $\alpha \in[0, \pi], A=\left\{e_{1}^{\alpha}\right\}$ is a smooth submanifold (with boundary). Clearly, by choice of $\delta_{4}, \gamma^{\alpha}$ is also free of conjugate points for each $\alpha$. This, using the fact that $T_{\delta_{3}}\left(\gamma_{1}^{\alpha}\right)$ (in the hd metric on $\operatorname{Geo}(S))$ is open, implies that $L\left[\gamma^{\alpha}\right]$ is a smooth function of $\alpha$. It is therefore reasonable to compute $(d / d \alpha) L\left[\gamma^{\alpha}\right]$.

The variation vector field for the variation $\left\{\gamma^{\alpha}\right\}$ is zero at $e_{2}$ and tangent to $A$ at $e_{1}^{\alpha}$. Since $\gamma_{1}^{\alpha \prime}\left(e_{1}^{\alpha}\right)$ is orthogonal to $A$ and $\Varangle\left(\gamma_{1}^{\alpha}, \gamma^{\alpha}\right)\left(e_{1}^{\alpha}\right)<\pi$ for $\alpha \in(0, \pi)$ (by choice of $\left.\delta_{4}\right), \gamma^{\alpha}$ is never orthogonal to $A$ for $\alpha \in(0, \pi)$. Thus, using the first variation formula, we see that $(d / d \alpha) L\left[\gamma^{\alpha}\right] \neq 0$. But it is quite clear that

$$
L\left[\gamma^{0}\right]=\left|L\left[\gamma_{1}\right]-L\left[\gamma_{2}\right]\right|<L\left[\gamma_{1}\right]+L\left[\gamma_{2}\right]=L\left[\gamma^{\pi}\right]
$$

so that $(d / d \alpha) L\left[\gamma^{\alpha}\right]>0$.

\subsection{Hinges in branched coverings of flattening surfaces}

If $S$ is a flattening surface of revolution with vertex $p, S^{*}$ will denote the infinite-sheeted branched covering space of $S$, branched over $p$, with the induced geometry. For computational purposes we may view $S^{*}$ in the following manner.

Suppose $\mathbf{R}^{2}$ is represented in polar coordinates $(r, \theta)$, and let $H=[0, \infty) \times$ R. Define $\pi_{0}: H \rightarrow \mathbf{R}^{2}$ by

$$
\pi_{0}(x, y)=(x, y \bmod 2 \pi)
$$

and topologize $H$ so that $\pi_{0}$ is continuous. Now consider the diagram

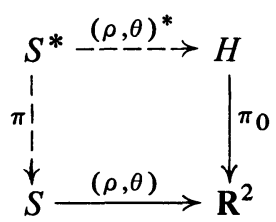


where $S^{*}$ is the $(\rho, \theta)$-pullback of $H$. Let the geometry of $S^{*}$ be the $\pi$-pullback of the geometry on $S$, and $p^{*}=\pi^{-1}(p)$. It is clear that the topology on $S^{*}$ which is induced by the geometry is the same as the topology which $S^{*}$ inherits as a pullback of $H$. Note that $S^{*}$ is not geodesically complete at $p^{*}$, but that otherwise geodesics are simply lifts of geodesics in $S$. Note also that minimal paths in $S^{*}$ are either lifts of minimal geodesics in $S$, or else can be written as $\mu_{1} \vee \mu_{2}$ where $\mu_{1}$ and $\mu_{2}$ are meridian segments in $S^{*}, \mu_{1}$ ending and $\mu_{2}$ beginning at $p^{*}$.

Let $\rho^{*}=(\rho, \theta)^{*}$ followed by projection into the first factor of $H$, and $\theta^{*}=(\rho, \theta)^{*}$ followed by projection into the second factor of $H$, and note that as a map from $S^{*}$ to $H,\left(\rho^{*}, \theta^{*}\right)=(\rho, \theta)^{*}$. Finally, if $\gamma \in \operatorname{Geo}\left(S^{*}\right)$, let $\theta_{\gamma^{*}}=\theta_{(\pi \circ \gamma)}$

3.3.1. Lemma. On $S^{*} \backslash\left\{p^{*}\right\}$

(1) $\left(\rho^{*}, \theta^{*}\right)$ is one to one, and hence a homeomorphism between $S^{*} \backslash\left\{p^{*}\right\}$ and $H \backslash(\{0\} \times \mathbf{R})$,

(2) each simple closed path determines a bounded and an unbounded component in $S^{*}, p^{*} \notin$ bounded component, and

(3) there are no closed geodesics.

Proof. (1) Since $(\rho, \theta)$ is one to one, so is $\left(\rho^{*}, \theta^{*}\right)$. Since $\pi$ and $(\rho, \theta)$ are open maps, so is $\left(\rho^{*}, \theta^{*}\right)$.

(2) This is standard, using the homeomorphism of (1).

(3) Suppose $\gamma:[0, b] \rightarrow S^{*}, \gamma \in \operatorname{Geo}(x, x)$. Then using (2) it is clear that $\theta_{\gamma^{*}}(0)=\theta_{\gamma^{*}}(b)$, which is impossible since $\theta_{\gamma^{*}}\left(=\theta_{(\pi \circ \gamma)}\right)$ is one to one. q.e.d.

Let $S_{+}^{*}$ denote some connected component of $\pi^{-1}\left(S_{+}\right)$. The following lemma along with Lemma 3.3 .5 provide the primary motivation for working in $S^{*}$ rather than in $S$. This will be discussed further in $\$ 4$.

3.3.2. Lemma. If $x \in S_{+}^{*}$ and $y \in S_{+}^{*}$, then $\operatorname{Geo}(x, y)$ contains precisely one element, and $\operatorname{Geo}(x, y) \subset \operatorname{Geo}\left(S_{+}^{*}\right)$.

Proof. That there is some $\gamma \in \operatorname{Geo}(x, y), \gamma \subset S_{+}^{*}$, is clear since the geometry on $S^{*}$ is the $\pi$-pullback of the geometry on $S$, and the minimal geodesic between $\pi(x)$ and $\pi(y)$ remains in $S_{+}$. That $\operatorname{Geo}(x, y) \cap \operatorname{Geo}\left(S_{+}^{*}\right)$ contains only one element follows immediately from Theorem 2.2.5 which describes the cut locus on $S$. If $\eta \in \operatorname{Geo}(x, y), \eta \neq \gamma$, then it must leave $S_{+}^{*}$. But Lemma 3.3.1(2) then implies that $\theta_{\eta}^{*}$ is not one to one. q.e.d.

Much of the technical difficulty which we will encounter throughout the remainder of this paper is due to the fact that $S^{*}$ is not complete. This next lemma provides us with the degree of completeness needed however. Note that we continue to apply the notation of $\$ 2.1$ to $S^{*}$ whenever the meaning is clear. 
3.3.3. Lemma. If $x \in S^{*}, y \in S^{*}, \gamma \in \operatorname{Geo}(x, y)$, and $\gamma$ lies below $z \in S^{*}$, then $\operatorname{Geo}(x, z) \neq \varnothing$. If $\gamma$ is also a minimal curve, so that $L[\gamma]=d(x, y)$, then there exists a minimal geodesic from $x$ to $z$.

Proof. Suppose $z \neq y$ and $\gamma$ is not contained in a meridian (in either case the lemma is obvious).

Let $\mu$ denote the meridian through $z$, and $\nu$ the meridian through $x$ with $\nu(0)=p^{*}$. See Fig. 3. Let $\varphi=\Varangle(\gamma, \nu)(x)$. Define a family of geodesics $\left\{\gamma_{\alpha}\right\}$, $\alpha \in[0, \varphi]$, each geodesic of which begins at $x$, lies above $\gamma$ in some neighborhood of $x$, and such that $\Varangle\left(\gamma, \gamma_{\alpha}\right)(x)=\alpha$. Since, for $\alpha \neq \varphi, \gamma_{\alpha}$ is not a meridian, each $\gamma_{\alpha}$ can be extended indefinitely and in particular, at least for small $\alpha$, until $\gamma_{\alpha}$ crosses $\mu$. Furthermore, it is clear that $\gamma_{\alpha} \cap \mu$ depends continuously on $\alpha$, and thus $A=\left\{\gamma_{\alpha} \cap \mu \mid \alpha \in[0, \varphi]\right\}$ is a connected set.

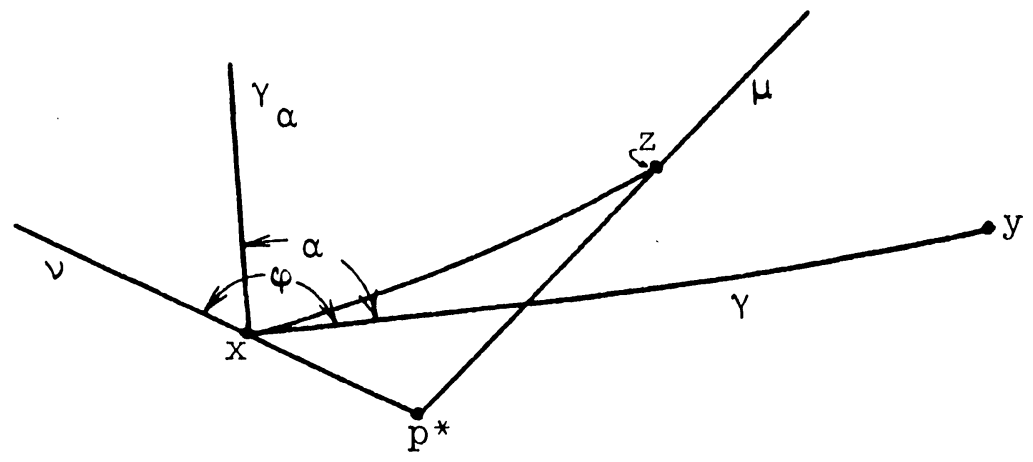

FIG. 3

It is clear that $\gamma_{\varphi} \cap \mu$ is empty and, by the continuous dependence of $\gamma_{\alpha}$ on $\alpha$, that $C=\left\{\alpha \mid \gamma_{\alpha} \cap \mu=\varnothing\right\}$ is closed. Let $a_{0}$ be the smallest element in $C$, and let $\left\{\alpha_{i}\right\}$ be a sequence of real numbers, $0<\alpha_{i}<\alpha_{i+1}<a_{0}$, which converge to $a_{0}$. Let $m_{i}=\gamma_{\alpha_{i}} \cap \mu$, and we claim that $\rho^{*}\left(m_{i}\right) \rightarrow \infty$ : if not, $m_{i} \rightarrow m \in \mu$; but again by continuous dependence of $\gamma_{\alpha}$ on $\alpha$, we see that $\gamma_{a_{0}} \cap \mu=m$, a contradiction.

Thus, since $\rho^{*}(\gamma \cap \mu)<\rho^{*}(z)$ and $A$ is connected, $\gamma_{\alpha} \cap \mu=z$ for some $\alpha \in[0, \varphi]$.

If $\gamma$ is a minimal geodesic, let $c$ be any minimal path from $x$ to $z$. If $c$ passes through $p^{*}$, then, since $\gamma$ lies below $z$ and meridians are the shortest paths from $p^{*}, c$ will cross $\gamma$. Since $z \neq y$, either $c$ or $\gamma$, or both, must continue to minimize beyond $c \cap \gamma$. But it is a standard fact that this cannot happen.

Thus the minimal path from $x$ to $z$ cannot pass through $p^{*}$, and so it must be a geodesic. That $\gamma$ lies below this minimal geodesic is also quite easy to see. q.e.d 
For $x \in S^{*}$ we call $y$ a cut point of $x$ if there is a geodesic from $x$ to $y$ which is minimal to, but not beyond, $y . C(x)$ will denote the collection of all cut points of $x$, and note that $C(x)$ may well be not-closed and/or disconnected. For example, let $S$ be a semi-infinite cylinder capped with a hemisphere (Example 2.3.2 with $\theta=\pi / 2$ ) take out the vertex, and let $x$ lie in the interior of the hemisphere. Then $C(x)=\{p\} \cup \bar{\mu}_{x} \mid(a, \infty)$, where $a \neq 0$. $\bar{\mu}_{x}(a) \notin C(x)$ since $x$ and $\bar{\mu}_{x}(a)$ are conjugate on $S$ only along a geodesic through $p$. The same problem arises in $S^{*}$.

The following lemma is the natural extension of the corresponding fact on complete manifolds.

3.3.4. Lemma. Let $\gamma \in \operatorname{Geo}\left(S^{*}\right)$. Then $\gamma\left(t_{0}\right)$ is a cut point of $x=\gamma(0)$ if and only if one of the following holds for $t=t_{0}$, and none holds for any smaller value of $t$ :

(1) $\gamma\left(t_{0}\right)=p^{*}$

(2) $x$ and $\gamma\left(t_{0}\right)$ are conjugate along $\gamma$, or

(3) there exists $\eta \in \operatorname{Cur}\left(x, \gamma\left(t_{0}\right)\right), \eta \neq \gamma$, with $L[\eta]=L[\gamma]$.

Proof. Since, if $\gamma$ is not a meridian nearby geodesics are also not meridians, the proof of the corresponding fact for complete manifolds, (see [2, Lemma 5.2]) applies. The only difference being that perhaps $\eta$ passes through $p^{*}$, and is thus only necessarily piecewise geodesic.

3.3.5. Lemma. Let $x \in S^{*}$ and $y \in C(x) \backslash\left\{p^{*}\right\}$. Then $d(x, y)>d\left(x, p^{*}\right)$ $+2 \delta_{1}$, where $\delta_{1}$ is chosen for the surface $S$ with $S^{*}=\pi^{*}(S)$ and $R \gg d(x, y)$.

Proof. Let $\gamma \in \operatorname{Geo}_{m}(x, y)$. If $x$ and $y$ are conjugate along $\gamma$, then $\pi(x)$ and $\pi(y)$ are conjugate along $\pi(\gamma)$. Thus, by Lemma 3.2.1, $L[\pi(\gamma)] \geqslant$ $d(\pi(x), p)+2 \delta_{1} ;$ and so $L[\gamma] \geqslant d\left(x, p^{*}\right)+2 \delta_{1}$.

Otherwise there exists $\eta \in \operatorname{Cur}(x, y), L[\eta]=L[\gamma]$. But then $\pi(\eta)$ and $\pi(\gamma)$ are distinct elements of $\operatorname{Cur}(\pi(x), \pi(y))$ of the same length, and thus $\pi(\gamma)$ has quit minimizing prior to $\pi(y)$. Thus, by Lemma 5.6 of [2] and Lemma 3.2.1, we once again see that $L[\gamma] \geqslant d\left(x, p^{*}\right)+2 \delta_{1}$.

3.3.6. Remark. Note that Lemma 3.2.1 parts (1), (2) and (3), with obvious minor modifications, apply to $S^{*}$ as well as to $S$. The incompleteness of $S^{*}$ prevents (5) from being applied in $S^{*}$. Likewise Lemma 3.2.2 applies to hinges in some $S_{+}^{*}$, but Lemma 3.2.3 does not apply to $S^{*}$. Thus we need the following lemma on $S^{*}$.

3.3.7. Lemma. Let $\left\{\left(\gamma_{1}^{\alpha}, \gamma_{2}, \alpha\right)\right\}, \alpha \in[a . b]$, be a family of hinges in $S^{*}$. Suppose

(1) $L\left[\gamma_{1}\right] \leqslant d\left(e_{0}, p^{*}\right)$

(2) $\gamma_{2} \subset S_{+}^{*}, S_{+}^{*}$ some given component of $\pi^{-1}\left(S_{+}\right)$, and 
(3) for each $\alpha \in[a, b]$ and each $t \in\left[0, b_{1}\right]$ there exists a geodesic from $\gamma_{1}^{\alpha}(t)$ to $e_{2}$ without cut points.

Then $(d / d \alpha)\left(d\left(e_{1}^{\alpha}, e_{2}\right)\right)>0$ for $\alpha \in(a, b)$.

Proof. Let $\gamma^{\alpha, t}$ denote the unique minimal geodesic from $\gamma_{1}^{\alpha}(t)$ to $e_{2}$ whose existence is assumed. From supposition (1) and Lemma 3.3.5 it follows that $A_{t}=\left\{\gamma_{1}^{\alpha}(t)\right\}, \alpha \in[a, b]$, is a smooth submanifold for each $t \in\left[0, b_{1}\right]$. Since $\gamma_{2}$ is in $S_{+}^{*}$ and hence unique between its end points (Lemma 3.3.2), $\left(\gamma^{\alpha, t}\right)^{\prime}(0)$ is not orthogonal to $A_{t}$ lest $\gamma_{1}^{\alpha} \mid[0, t] \vee \gamma^{\alpha, t}$ be another geodesic from $e_{0}$ to $e_{2}$. Furthermore, since $e_{2}$ has no cut points in $\left\{A_{t}\right\}, t \in\left[0, b_{1}\right]$, except perhaps $p^{*}$, it follows that $L\left[\gamma^{\alpha, t}\right]$ is a smooth function of both $\alpha$ and $t$. Thus, applying the first variation formula as in Lemma 3.2.3, $(\partial / \partial \alpha) L\left[\gamma^{\alpha, t}\right] \neq 0$.

It is easy to see that $\operatorname{sgn}\left((\partial / \partial \alpha) L\left[\gamma^{\alpha, t}\right]\right)$ is independent of $t$, and thus it suffices to prove the lemma for $t$ small. But if $t$ is sufficiently small the entire family $\left\{\gamma_{1}^{\alpha}\right\}, \alpha \in[0, \pi]$, is contained in $S_{+}^{*}$, in which case the lemma is apparent (cf. Lemma 3.2.2).

3.3.8. Remarks. (1) The geodesics required in Lemma 3.3.7(3) will always exist if $\gamma_{1}^{\alpha} \in S_{+}^{*}$ or $\gamma_{1}^{\alpha}$ lies below $e_{2}$ (Lemmas 3.3.2 and 3.3.3). The uniqueness is insured if $\left\{\gamma_{1}^{\alpha}\right\} \subset S_{+}^{*} \cup T_{d\left(e_{2}, p^{*}\right)+\delta_{1}}\left(e_{2}\right)$ (Lemmas 3.3.2 and 3.3.5).

(2) Note that in Lemma 3.3.7, $\gamma_{2}$ was allowed to be fairly long while in Lemma 3.2.3, $\gamma_{2}$ was required to be very short. Note however that in Lemma 3.2.3 we need not be as restrictive as in Lemma 3.3.7 to insure the existence of the $\gamma^{\alpha}$.

\section{GLOBAL COMPARISON THEOREMS}

Toponogov's theorem ([18]; see [2] for proof in English) is a beautiful and powerful global generalization of the Rauch comparison theorem. It gives distance estimates on a Riemannian manifold $M$ by comparison with a surface of constant curvature. Specifically,

Theorem (Toponogov). Let $M$ be a complete manifold with $K(x)>H \in \mathbf{R}$ for each $x \in M$. Let $\left(\gamma_{1}, \gamma_{2}, \alpha\right)$ be a hinge in $M$ with $\gamma_{1}$ minimal and, if $H>0$, $L\left[\gamma_{2}\right] \leqslant \pi / \sqrt{H}$. Let $\left(\bar{\gamma}_{1}, \bar{\gamma}_{2}, \alpha\right)$ be a hinge in the simply connected surface of constant curvature $H$ with $L\left[\bar{\gamma}_{1}\right]=L\left[\gamma_{i}\right]$, and $\bar{\gamma}_{i} \in \operatorname{Geo}\left(\bar{e}_{0}, \bar{e}_{i}\right)$. Then $d\left(e_{1}, e_{2}\right) \leqslant$ $d\left(\bar{e}_{1}, \bar{e}_{2}\right)$.

This theorem has provided estimates adequate for many important applications. It does, however, appear to be unnecessarily restrictive in the case of an open nonnegatively curved manifold. Since on any such manifold the curvature must come arbitrarily near zero (Bonnet's theorem), the comparison surface must be flat or negatively curved. If, for example, $M$ is a paraboloid 
and the hinge $\left(\gamma_{1}, \gamma_{2}, \alpha\right)$ has its vertex $e_{0}$ at the vertex of $M$, it is obvious that distance estimates on $\mathbf{R}^{2}$ will not be very accurate.

A natural question is: Can we improve the estimates on such a manifold? In the following we will show that we can, and that in fact, instead of a surface of constant curvature, an appropriate flattening surface of revolution may be used for the comparison surface. We actually prove two very similar such generalizations, the first serving as a lemma for the second.

\subsection{Definitions and notation}

Notational conventions established here will be used throughout this section without further comment.

4.1.1. Let $M^{n}$ denote a complete, open, nonnegatively curved manifold, let $\delta \geqslant 0$ and $p \in M$. Let $\bar{M}$ denote a flattening surface of revolution with vertex $\bar{p}$. We say that $M$ and $\bar{M}$ are $\delta$-correspondent at $p$ if, whenever $d(\bar{p}, \bar{x})+\delta \geqslant d(p, x)$, then $K(\bar{x}) \leqslant K(x)$, where $x \in M$ and $\bar{x} \in \bar{M}$. That is, the curvature in $\bar{M}$ falls off with respect to distance from $\bar{p}$ faster than the curvature in $M$ falls off with respect to distance from $p$. We will simply say that $M$ and $\bar{M}$ are correspondent at $p$ when we mean that $\delta=0$.

4.1.2. If $M$ and $\bar{M}$ are $\delta$-correspondent at $p$, and $\left(\gamma_{1}, \gamma_{2}, \alpha\right)$ is a hinge in $M$ with $e_{0}=p$ or $e_{1}=p$, then the hinge $\left(\bar{\gamma}_{1}, \bar{\gamma}_{2}, \alpha\right)$ in $\bar{M}$ given by specifying that

(1) $L\left[\bar{\gamma}_{i}\right]=L\left[\gamma_{i}\right]$, and

(2) $e_{i}=p \Rightarrow \bar{e}_{i}=\bar{p}$, where $\bar{\gamma}_{1} \in \operatorname{Geo}\left(\bar{e}_{0}, \bar{e}_{1}\right)$ and

$$
\bar{\gamma}_{2} \in \operatorname{Geo}\left(\bar{e}_{0}, \bar{e}_{2}\right) \text {, }
$$

is said to correspond to $\left(\gamma_{1}, \gamma_{2}, \alpha\right)$. Since $\bar{\gamma}_{1}$ is a segment of a meridian in $\bar{M}$ which includes as one end point the vertex $\bar{p}$ of $\bar{M}$, this correspondence is uniquely determined up to rotation and reflection of $\bar{M}$.

4.1.3. Given a hinge $\left(\gamma_{1}, \gamma_{2}, \alpha\right)$, we call a hinge $\left(g_{1}, g_{2}, a\right)$ a subhinge if $g_{2} \subset \gamma_{2}$. We say that the subhinge faces inward (outward) if $d\left(e_{2}, \varepsilon_{0}\right)<$ $(>) d\left(e_{2}, \varepsilon_{2}\right)$, where $g_{i} \in \operatorname{Geo}\left(\varepsilon_{0}, \varepsilon_{i}\right)$. See Fig. 4.

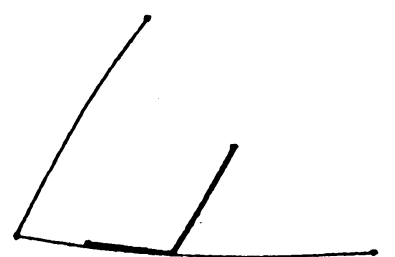

Inward Facing Subhinge

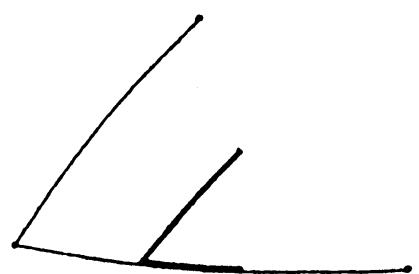

Outward Facing Subhinge

FIG. 4

For the remainder of this section, a hinge $\left(\gamma_{1}, \gamma_{2}, \alpha\right)$ in $M$ will be assumed to have either $e_{0}=p$. or $e_{1}=p$ and $\gamma_{1}$ minimal. A subhinge will always be a 
subhinge of such a hinge, with $p$ not necessarily an endpoint of one of the two geodesics making up the subhinge.

Let $h=\left(\gamma_{1}, \gamma_{2}, \alpha\right)$ and $\bar{h}=\left(\bar{\gamma}_{1}, \bar{\gamma}_{2}, \alpha\right)$ be corresponding hinges in $M$ and $\bar{M}$ respectively. We say that the subhinges $\left(\gamma_{1}, \gamma_{2}, \alpha\right)$ of $h$ and $\left(\bar{g}_{1}, \bar{g}_{2}, \alpha\right)$ of $\bar{h}$ are corresponding subhinges if:

(1) $L\left[g_{i}\right]=L\left[\bar{g}_{i}\right]$

(2) both face in the same direction, and

(3) $d\left(e_{0}, \varepsilon_{0}\right)=d\left(\bar{e}_{0}, \bar{\varepsilon}_{0}\right)$, where $\varepsilon_{0}$ is as above.

4.1.4. Let $h=\left(\gamma_{1}, \gamma_{2}, \alpha\right)$ be a hinge in $M$ and let $\Delta=\left(g_{1}, g_{2}, g_{3}\right)$ be a geodesic triangle in $M$ with $g_{2} \subset \gamma_{2}$. If $\left(\bar{\gamma}_{1}, \bar{\gamma}_{2}, \alpha\right)$ is a hinge in $\bar{M}$ which corresponds to $h$, we say that a geodesic triangle $\left(\bar{g}_{1}, \bar{g}_{2}, \bar{g}_{3}\right)$ in $\bar{M}$ corresponds to $\Delta$ if:

(1) $L\left[\bar{g}_{i}\right]=L\left[g_{i}\right]$

(2) $\bar{g}_{2} \subset \bar{\gamma}_{2}$, and

(3) $d\left(e_{0}, g_{2}(t)\right)=d\left(\bar{e}_{0}, \bar{g}_{2}(t)\right)$ as measured along $\gamma_{2}$ and $\bar{\gamma}_{2}$ respectively.

Finally, note that all of the above notions still make sense if we are working in $\bar{M}^{*}$ instead of $\bar{M}$. The only situation which demands any care occurs when $\left(\gamma_{1}, \gamma_{2}, \alpha\right)$ is a hinge in $\bar{M}^{*}$ with $e_{0}=p^{*}$. Then one must be sure that $\gamma_{1}$ and $\gamma_{2}$ lie on the same branch of $\bar{M}^{*}$.

In the following lemma we assume that $M$ and $\bar{M}$ are $\delta$-correspondent at $p \in M$, and that $\left(\gamma_{1}, \gamma_{2}, \alpha\right)$ and $\left(\bar{\gamma}_{1}, \bar{\gamma}_{2}, \alpha\right)$ are hinges (or subhinges) in $M$ and $\bar{M}$ respectively with $L\left[\gamma_{i}\right]=L\left[\bar{\gamma}_{i}\right]$. In this instance we do not assume that $e_{i}=p$ for $i=0$ or 1 .

4.1.5. Lemma. Suppose that $\left(\gamma_{1}, \gamma_{2}, \alpha\right)$ and $\left(\bar{\gamma}_{1}, \bar{\gamma}_{2}, \alpha\right)$ are as above with $0<\theta_{0}<\alpha<\pi-\theta_{0}$. Choose $\delta_{5}$ with $\varphi=\theta_{0}$ and $\varepsilon<\min \left\{\delta / 2, \delta_{2}\right\}$, and suppose that $L\left[\gamma_{2}\right]<\delta_{5}$. Further suppose that $d\left(\gamma_{1}(t), p\right)<d\left(\bar{\gamma}_{1}(t), p\right)$, and let $\bar{\gamma}$ denote the unique geodesic from $\bar{e}_{2}$ to $\bar{e}_{1}$ whose hd distance from $\bar{\gamma}_{1}$ is less than $\delta_{3}$. Then $L[\bar{\gamma}] \geqslant d\left(e_{2}, e_{1}\right)$.

Proof. It is quite straightforward to check that the hypotheses of Corollary 1.3.2 are satisfied.

Remarks. (1) Notice that if $e_{1}=p$ and $d\left(\bar{e}_{0}, \bar{p}\right) \geqslant d\left(e_{0}, p\right)$, then the hypotheses of the lemma are satisfied. (2) This of course works as well in $\bar{M}^{*}$ as long as the necessary geodesics exist. Rather than check this, we occasionally make the measurement in $M$ and then pull back up to $\bar{M}^{*}$.

\subsection{Two global comparison theorems}

In both Theorem 4.2.1 and Theorem 4.2.2, $M^{n}$ will denote a complete open nonnegatively curved $n$-dimensional Riemannian manifold, and $\bar{M}$ a flattening surface of revolution such that $M$ and $\bar{M}$ correspond at $p \in M$. 
4.2.1. Theorem Let $\left(\gamma_{1}, \gamma_{2}, \alpha_{0}\right)$ and $\left(\bar{\gamma}_{1}^{*}, \bar{\gamma}_{2}^{*}, \alpha_{0}\right)$ be corresponding hinges in $M$ and $\bar{M}^{*}$ respectively with $\gamma_{1}$ minimal, $e_{1}=p$ and $\bar{\gamma}_{2}{ }^{*} \subset \bar{M}_{+}{ }^{*}$, assume that $\bar{\gamma}_{i}^{*} \in \operatorname{Geo}\left(\bar{e}_{0}^{*}, \bar{e}_{i}^{*}\right)$. Let $\gamma_{2} \in \operatorname{Geo}_{m}\left(e_{2}, e_{1}\right)$. Then:

(A) $d\left(e_{1}, e_{2}\right) \leqslant d\left(\bar{e}_{1}^{*}, \bar{e}_{2}^{*}\right)$, and

(B) there exists a triangle $\left(c_{1}, \bar{\gamma}_{2}^{*}, c_{3}\right)$ in $\bar{M}^{*}$ which corresponds to $\left(-\gamma_{1}\right.$, $\left.\gamma_{2}, \gamma_{3}\right)$, with

$$
\begin{gathered}
\Varangle\left(-c_{1}, \bar{\gamma}_{2}^{*}\right)\left(\bar{e}_{0}^{*}\right) \leqslant \Varangle\left(\gamma_{1}, \gamma_{2}\right)\left(e_{0}\right), \\
\Varangle\left(\bar{\gamma}_{2}{ }^{*}, c_{3}\right)\left(\bar{e}_{2}{ }^{*}\right) \leqslant \Varangle\left(\gamma_{2}, \gamma_{3}\right)\left(e_{2}\right) .
\end{gathered}
$$

4.2.2. Theorem. Let $\left(\gamma_{1}, \gamma_{2}, \alpha_{0}\right)$ and $\left(\bar{\gamma}_{1}, \bar{\gamma}_{2}, \alpha_{0}\right)$ be corresponding hinges in $M$ and $\bar{M}$ respectively with $\gamma_{1}$ minimal and $e_{0}=p$. Let $\gamma_{3} \in \operatorname{Geo}_{m}\left(e_{2}, e_{1}\right)$. Then:

(A) $d\left(e_{1}, e_{2}\right) \leqslant d\left(\bar{e}_{1}, \bar{e}_{2}\right)$, and

(B) there exists a unique triangle $\left(c_{1}, \bar{\gamma}_{2}, c_{3}\right)$ in $\bar{M}$ which corresponds to $\left(-\gamma_{1}, \gamma_{2}, \gamma_{3}\right)$, with

$$
\begin{gathered}
\Varangle\left(-c_{1}, \bar{\gamma}_{2}\right)\left(\bar{e}_{0}\right) \leqslant \Varangle\left(\gamma_{1}, \gamma_{2}\right)\left(e_{0}\right), \\
\Varangle\left(\bar{\gamma}_{2}, c_{3}\right)\left(\bar{e}_{2}\right) \leqslant \Varangle\left(\gamma_{2}, \gamma_{3}\right)\left(e_{2}\right) .
\end{gathered}
$$

Proof. The proofs of these two theorems are quite similar, and will be given simultaneously. The proof is divided into a number of steps each of which is further broken down into discussions of the difficulties particular to either Theorem 4.2.1 or Theorem 4.2.2.

Fix $R \in \mathbf{R}, R>2\left(L\left[\gamma_{1}\right]+L\left[\gamma_{2}\right]\right)$.

(1) We will make several simplifying assumptions, and then later show that the theorems as stated are correct.

Assume that for some $\delta>0, M$ and $\bar{M}$ are $\delta$-correspondent. If $x \in \gamma_{2}$ and $\gamma_{x} \in \mathrm{Geo}_{m}(x, p)$, then assume that $\left\langle\gamma_{x}^{\prime}, \gamma_{2}^{\prime}\right\rangle(x) \in(-1,1)$. Note that, among other things, we have assumed that $\alpha_{0} \in(0, \pi)$.

(2) Notation. As usual, notation established either in $\bar{M}$ or $\bar{M}^{*}$ will actually apply to both by insertion or deletion of a star (*).

Let $A=\left\{\arccos \left(\left\langle\gamma_{2}{ }^{\prime}, \gamma_{x}{ }^{\prime}\right\rangle(x)\right) \mid x \in \gamma_{2}\right.$ and $\left.\gamma_{x} \in \operatorname{Geo}_{m}(p, x)\right\}$. Let $\theta_{s}=$ $\sup A$, and $\theta_{i}=\inf A$, as $x$ and $\gamma_{x}$ vary over all possibilities. Let $\theta_{m}=$ $\min \left(\theta_{i}, \pi-\theta_{s}\right)$, and note that $\theta_{m} \in(0, \pi / 2]$ by the assumptions of step (1) and the compactness of $\gamma_{2}$. Now choosen $\delta_{5}$ with $\varepsilon<\min \left\{\delta / 2, \delta_{2}\right\}$ and $\varphi=\theta_{m}$.

Let $\left\{x_{0}=e_{0}, x_{1}, \cdots, x_{n-1}, x_{n}=e_{2}\right\}$ partition $\gamma_{2}, x_{i}=\gamma\left(t_{i}\right)$, such that $t_{i}$ $<t_{i+1}$ and $d\left(x_{i}, x_{i+1}\right)<\delta_{5}$. Choose $\sigma_{i} \in \operatorname{Geo}_{m}\left(x_{i}, e_{1}\right)$ with $\sigma_{0}=\gamma_{1}$, and assume that $\sigma_{i}$ is parameterized on $\left[0, \lambda_{i}\right]$. Let $\left\{\bar{x}_{i}^{*}\right\}$ be a corresponding partition of $\bar{\gamma}_{2}^{*}$, with $\bar{x}_{i}^{*}=\bar{\gamma}_{2}^{*}\left(t_{i}\right)$. For $i<j$ define $\tau_{i, j}=\gamma_{2} \mid\left[t_{i}, t_{j}\right]$, and let $\bar{\tau}_{i, j}{ }^{*}$ denote the corresponding pieces of $\bar{\gamma}_{2}^{*}$. 
Call a geodesic $\sigma$ in $\bar{M}^{*}$, with $\sigma(0)=\bar{x}_{i}^{*}$, interior if for all $t$ sufficiently small the unique geodesic $\gamma^{*} \in \mathrm{Geo}_{m}\left(e_{0}^{*}, \sigma(t)\right)$ has $\Varangle\left(\gamma_{1}^{*}, \gamma^{*}\right)\left(e_{0}^{*}\right)<\alpha_{0}$. See Fig. 5. Let $\left\{\bar{\sigma}_{i}^{*}\right\}$ be a family of geodesics in $\bar{M}^{*}$ so that (i) $\bar{\sigma}_{i}^{*}(0)=x_{i}^{*}$, (ii) the subhinge $\left(\tau_{0, i}, \sigma_{i}, \beta_{i}\right)$ of $\left(\gamma_{1}, \gamma_{2}, \alpha_{0}\right)$ and the subhinge $\left(\bar{\tau}_{0, i}^{*}, \bar{\sigma}_{i}^{*}, \beta_{i}\right)$ of $\left(\bar{\gamma}_{1}^{*}, \bar{\gamma}_{2}^{*}, \alpha_{0}\right)$ are corresponding subhinges (note that this implicitly defines $\beta_{i}$ ), and (iii) so that each $\bar{\sigma}_{i}^{*}$ is an interior geodesic. Let $\alpha_{i}:=\pi-\beta_{i}$.
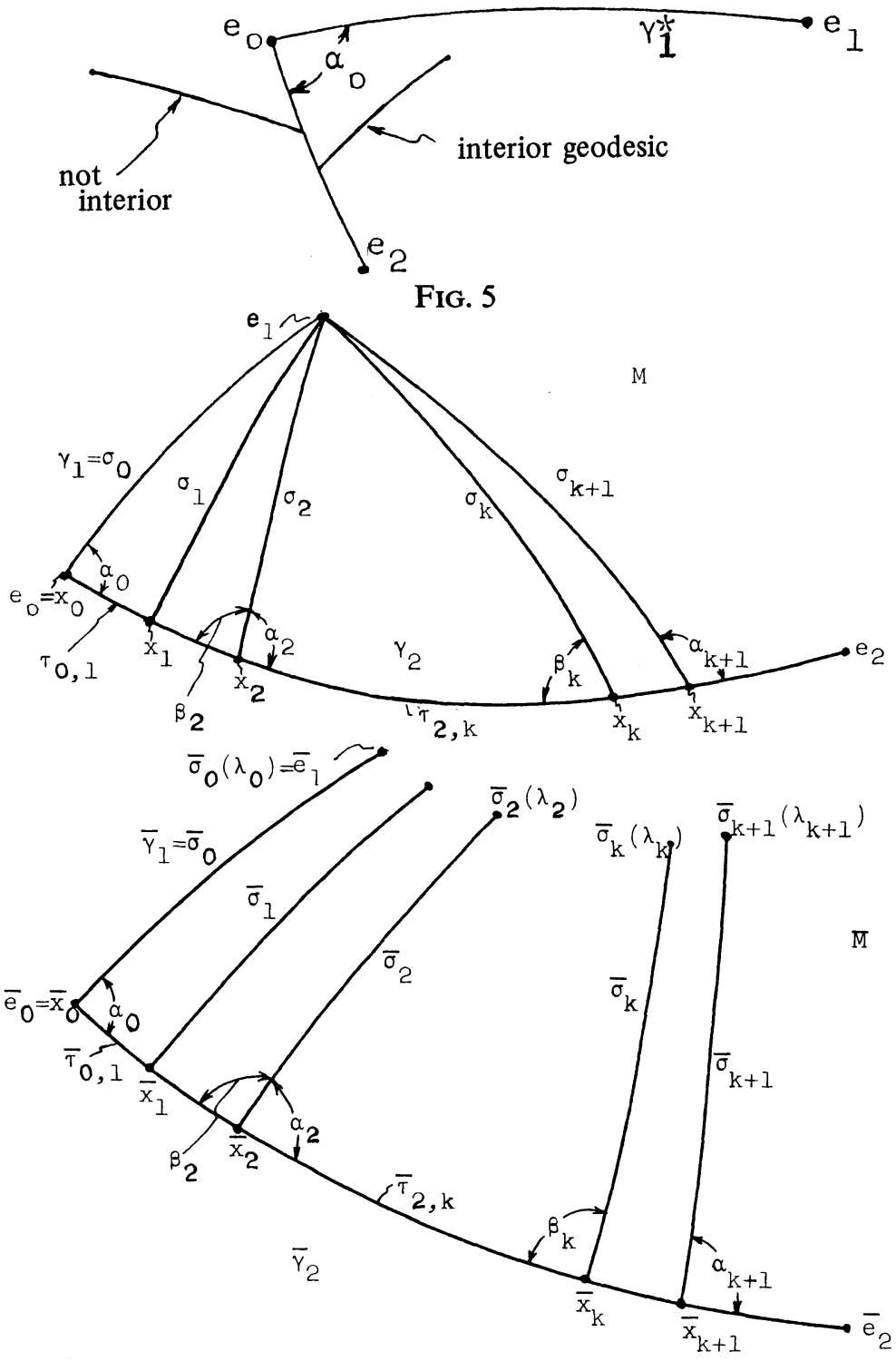

FIG. 6 
Finally, without loss of generality, assume in 4.2.1 that $\bar{\gamma}_{1}^{*} \in \partial \bar{M}_{+}{ }^{*}$ and $\bar{\gamma}_{2}{ }^{*} \in \bar{M}_{+}{ }^{*}$, and in Theorem 4.2.2 that $\bar{\gamma}_{2} \in \partial \bar{M}_{+}$and $\bar{\gamma}_{1} \in \bar{M}_{+}$. See Fig. 6.

(3) In addition to the assumption made in (1), also assume for Theorem 4.2.2 that the entire construction of part (2) is contained in $\bar{M}_{+}$. This is of course another simplifying assumption which must be dealt with later. Furthermore, we will postpone the proof of the uniqueness of the triangle in $B$ of Theorem 4.2.2 until step (11).

The proof will now proceed by induction.

(4) Let $\bar{g}_{0}$ be the unique geodesic from $\bar{x}_{1}$ to $\bar{e}_{1}$ with $\mathrm{hd}\left(\bar{g}_{0}, \bar{\sigma}_{0}\right)<\delta_{3}$. It follows from Lemma 4.1.5 that $L\left[\bar{g}_{0}\right] \geqslant d\left(e_{1}, x_{1}\right)$; and thus, in Theorem 4.2.1 since $\bar{g}_{0}$ is a meridian and in Theorem 4.2.2 since $\bar{g}_{0} \subset \bar{M}_{+}$, that $d\left(\bar{e}_{1}, \bar{x}_{1}\right) \geqslant$ $d\left(e_{1}, x_{1}\right)$. In Theorem 4.2.1 this estimate obviously applies on $\bar{M}^{*}$ as well. Hence (A) is true for the hinge $\left(\gamma_{1}, \tau_{0,1}, \alpha_{0}\right)$ in both Theorem 4.2.1 and Theorem 4.2.2.

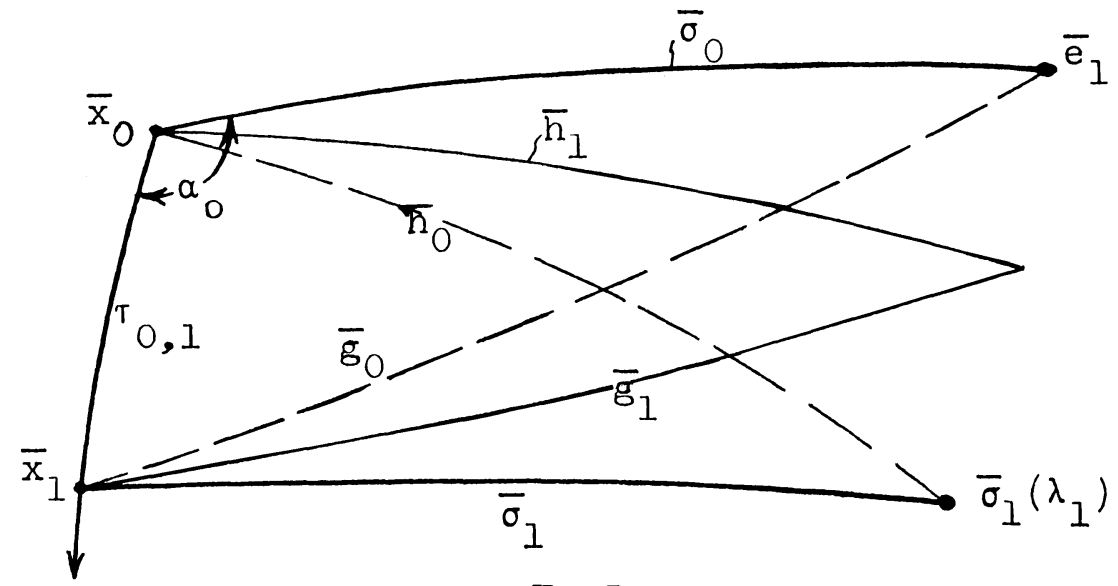

FIg. 7

(5) Let $\bar{h}_{0}$ be the unique geodesic from $\bar{x}_{0}$ to $\bar{\sigma}_{1}\left(\lambda_{1}\right)$ with $\mathrm{hd}\left(\overline{h_{0}}, \bar{\sigma}_{1}\right)<\delta_{3}$. We would now like to apply Lemma 4.1.5 to conclude that $L\left[\bar{h}_{0}\right]>d\left(e_{1}, x_{0}\right)$. To this end, note that: (i) for Theorem 4.2.1, the results of part (4) insure that the hypotheses of Lemma 4.1.5 are met; and (ii) for Theorem 4.2.2, the techniques of part (4) of Theorem 4.2.1 can be used to show that $d\left(\bar{p}, \bar{\sigma}_{i}(t)\right)>$ $d\left(p, \sigma_{i}(t)\right)$ for $t \in\left[0, \lambda_{1}\right]$. Thus $L\left[\bar{g}_{0}\right] \geqslant L\left[\bar{\sigma}_{1}\right]$ and $L\left[\bar{h}_{0}\right]>L\left[\bar{\sigma}_{0}\right]$ in both Theorem 4.2.1 and Theorem 4.2.2.

Now, using Lemma 3.2.3, decrease $\alpha_{0}$ by moving $\bar{\sigma}_{0}$ until $d\left(\bar{\sigma}_{0}\left(\lambda_{0}\right), \bar{x}_{1}\right)$, as measured along the unique nearly geodesic, is $L\left[\bar{\sigma}_{1}\right]$. Let $\bar{h}_{1}$ denote the geodesic resulting from this movement, and $\bar{g}_{1}$ be the unique nearby geodesic from $x_{1}$ to $\bar{h}_{1}\left(\lambda_{0}\right)$. We claim that the triangle $\left(-\bar{h}_{1}, \bar{\tau}_{0,1}, \bar{g}_{1}\right)$ corresponding to $\left(-\sigma_{0}, \tau_{0,1}, \sigma_{1}\right)$ is the one required in part (B). See Fig. 7. 
Since $\Varangle\left(\bar{h}_{1}, \tau_{0,1}\right)\left(x_{0}\right) \leqslant \alpha_{0}$ by construction, the only thing left to check is that $\Varangle\left(\bar{g}_{1},-\bar{\tau}_{0,1}\right)\left(\bar{x}_{1}\right) \leqslant \beta_{1}$. But it is quite easy to see that if $\beta_{1}$ is decreased by moving $\bar{\sigma}_{1}$ until $d\left(\bar{\sigma}_{1}\left(\lambda_{1}\right), \bar{x}_{0}\right)$, as measured along the nearby geodesic, is $L\left[\bar{\sigma}_{0}\right]$, then the resulting geodesic must be $\bar{g}_{1}$. Since $\bar{p}$ is not in the interior of the bounded region determined by $\left(-\bar{h}_{1}, \bar{\tau}_{0,1}, \bar{g}_{1}\right)$, the triangle, in the case of Theorem 4.2.1, can be lifted to $\bar{M}^{*}$.

Hence (B) is true for the triangle $\left(-\sigma_{0}, \tau_{0,1}, \sigma_{1}\right)$ in both Theorem 4.2.1 and Theorem 4.2.2.

Remark. In this step of Theorem 4.2.1 we had to work on $\bar{M}$ since there was no a priori guarantee that $\bar{h}_{0}$ could be lifted to $\bar{M}^{*}$.

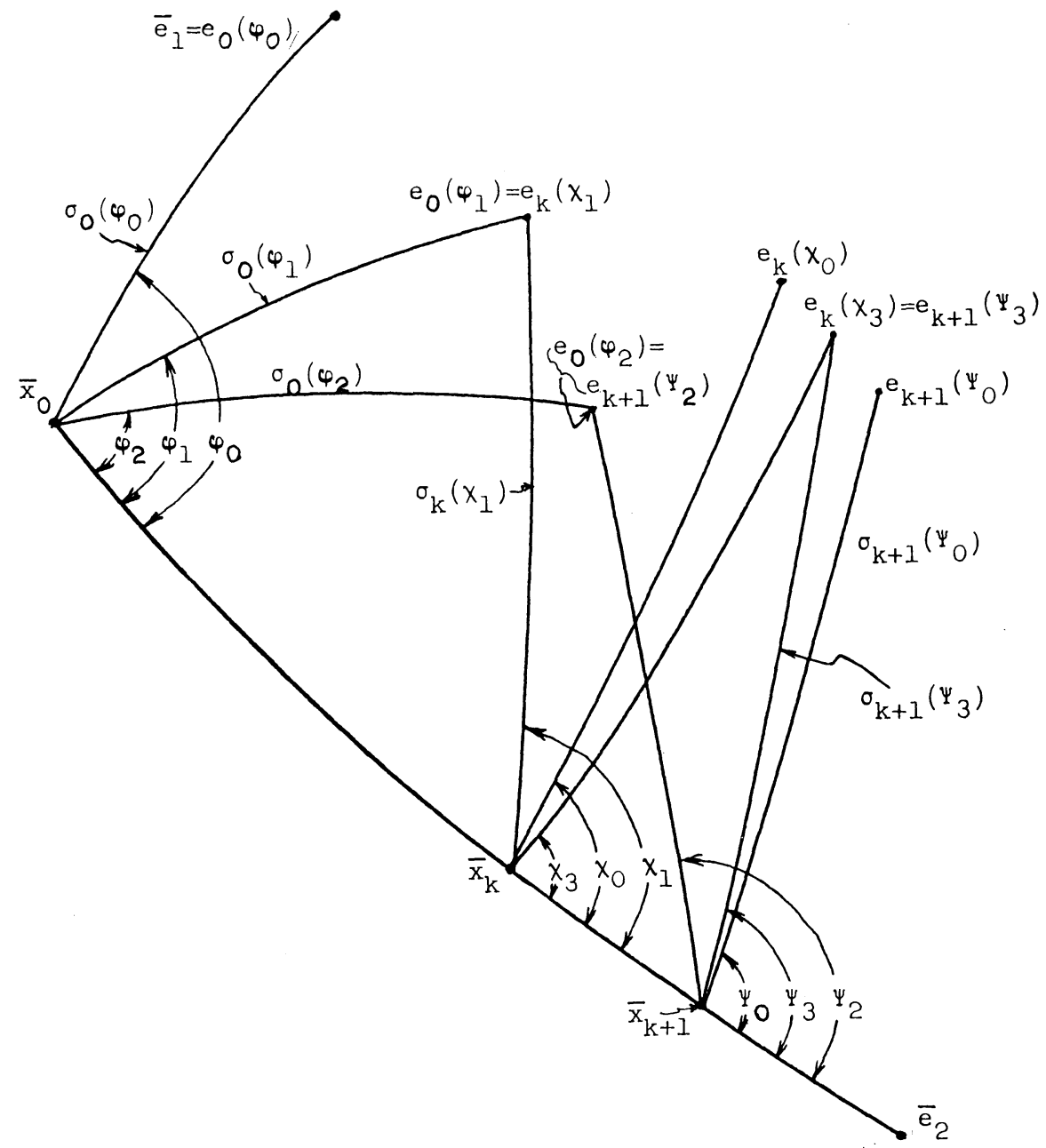

FIG. 8 
(6) Now suppose that $d\left(e_{0}, x_{k}\right) \leqslant d\left(\bar{e}_{0}, \bar{x}_{k}\right)$ and that (B) is true for the triangle $\left(-\sigma_{0}, \tau_{0, k}, \sigma_{k}\right)$. In the following two parts several families of hinges shall be used. To avoid a surfeit of subscripted superscripts, we introduce the following notation:

$$
\begin{aligned}
& \varphi_{0}=\alpha_{0}, \quad \chi_{0}=\alpha_{k}, \quad \Psi_{0}=\alpha_{k+1} ; \\
& \sigma_{0}(\varphi)=\bar{\sigma}_{0}^{\left(\varphi_{0}-\varphi\right)}, \quad \sigma_{k}(\chi)=\bar{\sigma}_{k}^{\left(\chi_{0}-\chi\right)}, \\
& c_{k+1}(\Psi)=\bar{\sigma}_{k+1}^{\left(\Psi_{0}-\Psi\right)} \quad \text { (cf. §3.1); } \\
& e_{0}(\varphi)=\sigma_{0}(\varphi)\left(\lambda_{0}\right), \quad e_{k}(\chi)=\sigma_{k}(\chi)\left(\lambda_{k}\right), \\
& e_{k+1}(\Psi)=\sigma_{k+1}(\Psi)\left(\lambda_{k+1}\right) \text {. }
\end{aligned}
$$

We consider $\bar{\gamma}_{2}$, and thus $\bar{\tau}_{i, j}$ for $0 \leqslant i<j \leqslant n$, to be fixed; so that increasing or decreasing angles $\varphi, \chi$, or $\Psi$ will be by moving the $\sigma_{i}$. Let $\left(-\sigma_{0}\left(\varphi_{1}\right), \bar{\tau}_{0, k}, \sigma_{k}\left(\chi_{1}\right)\right)$ be the triangle in $\bar{M}$ which corresponds to $\left(-\sigma_{0}, \tau_{0, k}, \sigma_{k}\right)$. Observe that $\varphi_{1}<\varphi_{0}$ and $\chi_{1}>\chi_{0}$ by the induction hypothesis. It may help to refer to Fig. 8. Then again, it may not.

Note that in Theorem 4.2.2 $\left(-\sigma_{0}\left(\varphi_{1}\right), \tau_{0, k}, \sigma_{k}\left(\chi_{1}\right)\right)$ is contained in $\bar{M}_{+}$, while in Theorem 4.2.1 we have no such assurance. This is one of the reasons why in Theorem 4.2.1 it is convenient to work in $\bar{M}^{*}$.

Since Theorem 4.2.1 will be used as a lemma for Theorem 4.2.2, it is necessary to first finish Theorem 4.2.1, and then come back to Theorem 4.2.2. Steps (7) and (8) refer only to Theorem 4.2.1.

(7) We will first show that as $\varphi$ is decreased from $\varphi_{0}$ to $\varphi_{1}$, the distance from $\sigma_{0}(\varphi)$ to $\bar{x}_{k+1}{ }^{*}$ is decreased. Specifically, since $\sigma_{0}\left(\varphi_{0}\right) \subset \bar{M}_{+}{ }^{*} \cup \bar{p}^{*}$, Lemma 3.3.7 implies that $\left.(d / d \varphi) d\left(e_{0}(\varphi), \bar{x}_{k+1}{ }^{*}\right)\right|_{\varphi=\varphi_{0}}>0$. This remains true, as $\varphi$ is decreased, so long as

$$
\sigma_{0}(\varphi) \subset\left(\bar{M}_{+}^{*} \cup T_{d\left(\bar{x}_{k+1}^{*}, \bar{p}^{*}\right)+\delta_{1}}\left(\bar{x}_{k+1}{ }^{*}\right)\right),
$$

and $\sigma_{0}(\varphi)$ is either in $\bar{M}_{+}{ }^{*}$ or below $\bar{x}_{k+1}{ }^{*}$. The latter condition clearly remains in force as $\varphi$ is decreased. To see that the former does also, suppose that

$$
e_{0}(\bar{\varphi}) \in \mathrm{Cl}\left(T_{d\left(\bar{x}_{k+1}^{*}, \bar{p}^{*}\right)}\left(\bar{x}_{k+1}^{*}\right)\right)
$$

for some $\bar{\varphi}$. But since the hypotheses of Lemma 3.3.7 are satisfied, we see that $d\left(e_{0}(\varphi), \bar{x}_{k+1}^{*}\right)$ continues to decrease, which forces $e_{0}(\varphi)$ to remain in $T_{d\left(\bar{x}_{k+1}{ }^{*}, \bar{p}^{*}\right)+\delta_{1}}\left(\bar{x}_{k+1}{ }^{*}\right)$. This of course applies as well to each point on $\sigma_{0}(\varphi)$, so that

$$
d\left(e_{0}\left(\varphi_{1}\right), \bar{x}_{k+1}^{*}\right) \leqslant d\left(e_{0}\left(\varphi_{0}\right), \bar{x}_{k+1}^{*}\right)=d\left(\bar{e}_{1}^{*}, \bar{x}_{k+1}^{*}\right)
$$


This same argument applied to $\sigma_{k}(\chi)$ implies that

$$
d\left(e_{0}\left(\varphi_{1}\right), \bar{x}_{k+1}{ }^{*}\right)=d\left(e_{k}\left(\chi_{1}\right), \bar{x}_{k+1}{ }^{*}\right) \geqslant d\left(e_{k}\left(\chi_{0}\right), \bar{x}_{k+1}{ }^{*}\right) .
$$

Now just as in step (4) we conclude that

$$
d\left(e_{k}\left(\chi_{0}\right), \bar{x}_{k+1}^{*}\right)>L\left[\sigma_{k+1}\right]=d\left(e_{1}, x_{k+1}\right) .
$$

Combining all inequalities yields

$$
d\left(\bar{e}_{1}^{*}, \bar{x}_{k+1}^{*}\right) \geqslant d\left(e_{1}, x_{k+1}\right),
$$

which is $(\mathrm{A})$.

(8) We will now show that (B) holds for $\left(\sigma_{0}, \tau_{0, k+1}, \sigma_{k+1}\right)$.

The techniques of step (5) show that (B) is true for the triangle $\left(-\sigma_{k}, \tau_{k, k+1}, \sigma_{k+1}\right)$. Let $\left(\sigma_{k}\left(\chi_{3}\right), \tau_{k, k+1}{ }^{*}, \sigma_{k+1}\left(\Psi_{3}\right)\right)$ denote the corresponding triangle in $\bar{M}^{*}$. Note that $\Psi_{3}>\Psi_{0}$, and that increasing $\Psi_{3}$ will bring $e_{k+1}(\Psi)$ still closer to $\bar{x}_{k}^{*}$.

Decrease $\varphi_{1}$ to $\varphi_{2}$, so that $d\left(e_{0}\left(\varphi_{2}\right), \bar{x}_{k+1}{ }^{*}\right)=L\left[\sigma_{k+1}\right]$. Clearly Lemma 3.3.7 continues to apply during this movement. Let $\Psi_{2}$ be the angle determined by the (unique) geodesic $\sigma_{k+1}\left(\Psi_{2}\right) \in \operatorname{Geo}\left(\bar{x}_{k+1}{ }^{*}, e_{0}\left(\varphi_{2}\right)\right)$.

As noted above, $\varphi_{2}<\varphi_{1}<\varphi_{0}$. To see that $\Psi_{2}>\Psi_{0}$, observe that $d\left(e_{0}\left(\varphi_{2}\right), \bar{x}_{k}^{*}\right)<L\left[\sigma_{k}\right]$, and thus (working in $\bar{M}$ if needed) $\Psi_{3}$ must be increased to bring $e_{k+1}(\Psi)$ nearer to $\bar{x}_{k}^{*}$; i.e., so that $d\left(e_{k+1}\left(\Psi_{2}\right), \bar{x}_{k}^{*}\right)<L\left[\sigma_{k}\right]$.

Hence $\mathrm{B}$ is true for $\left(\sigma_{0}, \tau_{0, k+1}, \sigma_{k+1}\right)$, and the proof of Theorem 4.2.1 is complete.

We now repeat steps (7) and (8) for Theorem 4.2.2, using Theorem 4.2.1 as needed.

(9) Since $\left(-\sigma_{0}\left(\varphi_{1}\right), \bar{\tau}_{0, k}, \sigma_{k}\left(\chi_{1}\right)\right) \subset \bar{M}_{+}$by the induction hypothesis, it is clear that $d\left(\bar{e}_{1}, \bar{x}_{k+1}\right) \geqslant d\left(e_{k}\left(\chi_{1}\right), x_{k+1}\right)$. Since $\sigma_{k}\left(\chi_{0}\right) \subset \bar{M}_{+}$, it is clear that $d\left(e_{k}\left(\chi_{1}\right), \bar{x}_{k+1}\right) \geqslant d\left(e_{k}\left(\chi_{0}\right), \bar{x}_{k+1}\right)$. Theorem 4.2.1, applied to the hinge $\left(\tau_{0, k}, \sigma_{k}, \beta_{k}\right)$, implies that $d\left(\sigma_{k}(t), p\right) \leqslant d\left(\bar{\sigma}_{k}(t), \bar{p}\right)$. Thus Lemma 4.1.5 implies that $d\left(e_{k}\left(\chi_{0}\right), \bar{x}_{k+1}\right) \geqslant d\left(e_{1}, x_{x+1}\right)$. Hence $d\left(\bar{e}_{1}, \bar{x}_{k+1}\right) \geqslant d\left(e_{1}, x_{k+1}\right)$.

(10) We again apply Theorem 4.2.2, this time to the hinge $\left(\tau_{0, k+1}, \sigma_{k+1}, \beta_{k+1}\right)$, and conclude that $d\left(\sigma_{k+1}(t), p\right) \leqslant d\left(\bar{\sigma}_{k+1}(t), \bar{p}\right)$. Thus Lemma 4.1.5 implies that $d\left(e_{k+1}\left(\Psi_{0}\right), \bar{x}_{k}\right) \geqslant L\left[\sigma_{k}\right]$. (B) now follows for the triangle $\left(-\sigma_{0}, \tau_{0, k+1}, \sigma_{k+1}\right)$ in a manner analogous to that in step (8). Uniqueness is discussed in step (11).

(11) Loose ends: First, the uniqueness of the triangle in (B) of Theorem 4.2.2 is clear since the triangle is contained in $\bar{M}_{+}$and has one vertex at $p$.

For the assumption in step (3), note that any flattening surface of revolution is a deformation of $\mathbf{R}^{2}$ through flattening surfaces. Suppose that $\left\{S^{t}\right\}$ is such a deformation with $S^{0}=\mathbf{R}^{2}$ and $S^{1}=\bar{M}$, and let $\gamma_{i}^{t}$ and $\sigma_{i}^{t}$ denote the geodesics on $S^{t}$ resulting from the construction in step (2). Let $B^{t}$ denote the 
region of $S_{+}^{t}$ which is bounded by $\mu_{e_{1}}^{t}$ and $\mu_{e_{2}}^{t}$. Steps (4)-(10) show that if $\sigma_{i}^{t}$ is in $S_{+}^{t}$, it is actually restricted to $B^{t}$. Since the $\sigma_{i}^{t}$ are continuous with respect to $t$, since no $\sigma_{i}^{t}$ passes through $p$, and since the $\sigma_{i}^{0}$ are clearly in $S_{+}^{0}$, it follows that the $\sigma_{i}^{1}$ are in $S_{+}^{1}=\bar{M}_{+}$. Here we are also using the fact that $\alpha_{0}<\pi$.

For the assumption in step (1) concerning $\delta$-correspondence, construct $\bar{M}$ corresponding to $M$. Since the theorem holds if the metric of $\bar{M}$ is multiplied by any constant greater than 1 , it must, by continuity, hold on $\bar{M}$ itself.

If for any $x,\left\langle\gamma_{x}{ }^{\prime}, \gamma_{2}{ }^{\prime}\right\rangle(x)=-1$ (see step (1)), then $\alpha_{0}=\pi$, and the theorem holds by continuity.

Again referring to the assumptions in step (1), suppose that $\bar{t} \in\left[0, b_{2}\right]$ is the smallest number such that $\left\langle\gamma_{y}{ }^{\prime}, \gamma_{2}{ }^{\prime}\right\rangle(y)=+1$, where $y=\gamma_{2}(\bar{t})$. Then, by continuity, the theorem holds for the hinge $\left(\gamma_{1}, \gamma_{2} \mid[0, \bar{t}], \alpha_{0}\right)$. since $\gamma_{2} \mid\left[\bar{t}, b_{2}\right]$ is contained in the minimal geodesic from $y$ to $e_{1}$, the theorem clearly holds for $\left(\gamma_{1}, \gamma_{2}, \alpha\right)$.

\section{MANIFOLDS DIFFEOMORPHIC TO EUCLIDEAN SPACE}

Conjecture (Cheeger and Gromoll). If $M^{n}$ is a complete open nonnegatively curved manifold, and all sectional curvatures at some point $x \in M$ are positive, then $M$ is diffeomorphic to $\mathbf{R}^{n}$.

This conjecture is known to be true if $n=2,3$ or 4; see [5], [3] and [4] respectively. If the words "some point" are replaced by "every point", the conjecture follows from [9] or [3].

In this section we apply Theorem 4.2 to the soul construction of Cheeger and Gromoll [3] to prove a weak version of this conjecture. In dimensions greater than 4, this is the first such result which does not require $M$ to have positive curvature everywhere off a compact set.

\subsection{The soul of a manifold}

We begin by recalling several facts concerning the above mentioned soul construction. $M^{n}$ will continue to denote a complete open nonnegatively curved manifold.

5.1.1. Definition. A set $C \subset M$ is said to be totally convex if for any $x, y \in C, \operatorname{Geo}(x, y) \subset \operatorname{Geo}(C)$.

5.1.2. Theorem (Cheeger and Gromoll). $M$ contains a compact totally geodesic submanifold $S$ without boundary which is totally convex, $0 \leqslant \operatorname{dim} S<$ $\operatorname{dim} \boldsymbol{M}$.

Proof. We merely outline part of the construction of the set $S$. For further details see Cheeger and Gromoll [3], or Cheeger and Ebin [2]. 
Let $x \in M$ and let $\gamma:[0, \infty) \rightarrow M$ be any geodesic ray beginning at $x$; i.e., $\gamma$ is a globally minimal geodesic. Let $B_{x}(\gamma)=\{y \in M \mid d(\gamma(t), y)<t, t \in$ $[0, \infty)\}$, and set $H_{x}(\gamma)=M \backslash B_{x}(\gamma)$. We call $H_{x}(\gamma)$ a complementary half space.

Define $C_{x}=\cap\left(H_{x}(\gamma)\right)$ as $\gamma$ ranges over all rays beginning at $x$. It is not difficult to show that $C_{x}$ is compact. $S$ is now constructed as a certain subset of $C_{x}$.

5.1.3. Definition. The set $S \subset M$ constructed in Theorem 5.1 .2 is called a soul of $M$.

5.1.4. Theorem (Cheeger and Gromoll). Let $S$ be a soul of $M$. Then $M$ is diffeomorphic to the normal bundle $\nu(S)$ of $S$ in $M$. Furthermore, if $K(x)>0$ for each $x \in S$, then $S$ is a point, and hence $M$ is diffeomorphic to $\mathbf{R}^{n}$.

Proof. See Cheeger and Gromoll [3] and either Poor [16] or Šarafutdinov [17].

\subsection{Compact half spaces and shriveled souls}

Theorem. Let $M^{n}$ be a complete open nonnegatively curved manifold. Suppose $p \in M$ and $\gamma$ is a ray starting at $p$.

5.2.1. If there is an $r_{1} \in \mathbf{R}$ such that for each $x \in T_{r_{1}}(p), K(x)>\left(\pi / 3 r_{1}\right)^{2}$, then $H_{x}(\gamma)$ is compact.

5.2.2. If there is an $r_{2} \in \mathbf{R}$ such that for each $x \in T_{r_{2}}(p), K(x)>\left(\pi / \lambda r_{2}\right)^{2}$, where $\lambda \cong 2.46057$, then $M$ is diffeomorphic to $\mathbf{R}^{n}$.

Proof. This is an easy application of Theorem 4.2.2 to the construction outlined in Theorem 5.1.2. We first need to construct appropriate comparison surfaces.

Let $C_{r}^{H}$ denote a cone with spherical cap of curvature $H$ and radius $r$. That is, $C_{r}^{H}$ is the surface of revolution generated by the curve $f: \mathbf{R}_{+} \rightarrow \mathbf{R}^{2}, f$ defined by

$$
f(t)=\left\{\begin{array}{lr}
\left(\frac{\sin (t)}{\sqrt{H}}, \frac{1-\cos (t)}{\sqrt{H}}\right), & 0 \leqslant t \leqslant \theta, \\
\left(\frac{\sin (\theta)}{\sqrt{H}}+(t-r) \sin (\theta), \frac{1-\cos (\theta)}{\sqrt{H}}+(t-r) \cos (\theta)\right), & t>\theta,
\end{array}\right.
$$

where $\theta=r \sqrt{H}$. See Fig. 9 (a).

If $x \in C_{r}^{H}$ and $y \in \bar{\mu}_{x}$, let $g$ denote a minimal geodesic from $x$ to $y$. Let $P$ denote the parallel through the point $\left(H^{-1 / 2} \cdot \sin (\theta)\right), H^{-1 / 2} \cdot(1-\cos (\theta)) \in$ $f \subset S$, and let $p=d(x, P), q=d(y, P)$. Let $s$ and $\varphi$ be as indicated in Fig. 9 (b). 

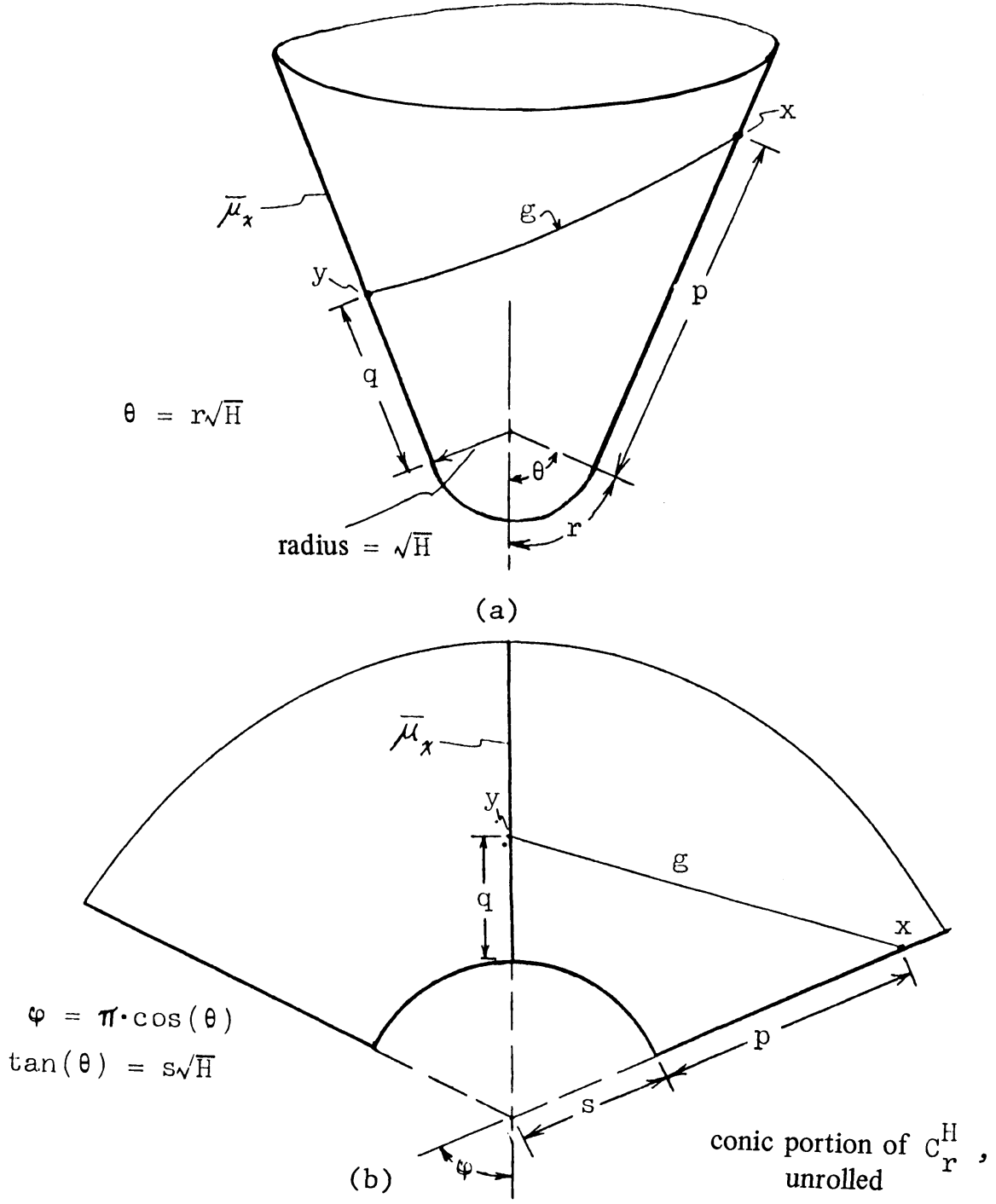

FIG. 9

We will now find conditions on $\theta$ which allow us to choose $x \in C_{r}^{H}$ so that $L[g]<p+r$ in the cases

(1) $g$ minimal between $x$ and $\bar{\mu}_{x}$, and

(2) $q=0$.

Case (1). $\quad L[g]=(p+s) \sin (\varphi)$.

We need conditions so that

$$
(p+s) \sin (\varphi)<(p+r),
$$


or equivalently,

$$
p(1-\sin (\varphi))+(r-s \cdot \sin (\varphi))>0 .
$$

Hence if $\sin (\varphi) \neq 1$, we may choose $p$ large enough to insure that the inequality holds. Thus we need $\varphi<\pi / 2$, or $\pi / 3<\theta \leqslant \pi / 2$; which is to say

$$
\frac{\pi}{3 \sqrt{H}}<r \leqslant \frac{\pi}{2 \sqrt{H}} \text {. }
$$

Case (2). $L[g]^{2}=s^{2}=(p+s)^{2}-2 s(p+s) \cdot \cos (\varphi)$.

We need conditions so that

$$
s^{2}+(p+s)^{2}-2 s(p+s) \cdot \cos (\varphi)<(p+r)^{2},
$$

i.e.,

$$
2 p[s \cdot \cos (\varphi)+r-s]+\left[r^{2}+2 s^{2} \cdot \cos (\varphi)-2 s^{2}\right]>0 .
$$

Hence if $s \cdot \cos (\varphi)+r-s>0$, we may choose $p$ large enough so that the inequality holds.

Thus the condition is

$$
\frac{\tan (\theta)}{\sqrt{H}} \cdot \cos (\varphi)+\frac{\theta}{\sqrt{H}}-\frac{\tan (\theta)}{\sqrt{H}}>0,
$$

i.e.,

$$
[\tan (\theta)] \cdot[\cos (\pi \cos (\theta))]+\theta-\tan (\theta)>0 .
$$

This is true for $\pi / \lambda<\theta \leqslant \pi / 2$, where $\lambda \cong 2.46057$. Thus we need

$$
\frac{\pi}{\lambda \sqrt{H}}<r \leqslant \frac{\pi}{2 \sqrt{H}} \text {. }
$$

Despite the fact that $C_{r}^{H}$ is not smooth, we may use it as a comparison surface by virtue of the previously mentioned approximation theorems in [1].

For Theorem 5.2.1, choose $H_{1} \in \mathbf{R}$ such that

$$
\left(\pi / 3 r_{1}\right)^{2} \leqslant H_{1} \leqslant K(x) \quad \forall x \in T_{r_{1}}(p),
$$

and for Theorem 5.2.2 choose $H_{2} \in \mathbf{R}$ such that

$$
\left(\pi / \lambda r_{2}\right)^{2} \leqslant H_{2} \leqslant K(x) \quad \forall x \in T_{r_{2}}(p) .
$$

Let $\eta$ be any minimal geodesic in $M$ which starts at $p$.

In Theorem 5.2.1, by comparison with $C_{r_{1}}^{H_{1}}$, there is some $t_{0}>0$ such that $\eta(t) \in B_{p}(\gamma)$ for $t>t_{0}$; and thus $H_{p}(\gamma) \subset T_{t_{0}}(p)$.

In Theorem 5.2.2, by comparison with $C_{r_{2}}^{\mathrm{H}_{2}}, \eta(t) \in B_{p}(\gamma)$ for $t>r_{2}$. Thus $S \subset H_{p}(\gamma) \subset T_{r_{2}}(p)$. But $K(x)>0$ for all $x$ in $T_{r_{2}}(p)$. Hence by Theorem 5.1.4, $M$ is diffeomorphic to $\mathbf{R}^{n}$. 


\section{References}

[1] A. D. Alexandrov \& V. A. Zalgaller, Intrinsic geometry of surfaces, Transl. Math. Monographs, Vol. 15, Amer. Math. Soc., 1967.

[2] J. Cheeger \& D. Ebin, Comparison theorems in Riemannian geometry, North Holland/ American Elsevier, Amsterdam-Oxford-New York, 1975.

[3] J. Cheeger \& D. Gromoll, On the structure of complete manifolds of nonnegative curvature, Ann. of Math. 96 (1972) 413-443.

[4] private communication.

[5] S. Cohn-Vossen, Kürzeste Wege und Totalkrümmung auf Flächen, Compositio Math. 2 (1935) 69-133.

[6] __, Totalkrümmung und geodätische Linien auf einfachzusammenhängenden offen vollständigen Flächenstücken, Rec. Math. Moscow 43 (1936) 139-163.

[7] G. Darboux, Lecons sur la theorie generale des surfaces, Livre VI, Gauthier-Villars et Fils, Paris, 1894.

[8] H. Gluck \& D. Singer, The existence of nontriangulable cut loci, Bull. Amer. Math. Soc. 82 (1976) 599-602.

[9] D. Gromoll \& W. Meyer, On complete open manifolds of positive curvature, Ann. of Math. 90 (1969) 75-90.

[10] W. Klingenberg, Eine Vorlesung über Differentialgeometrie, Springer, Berlin, 1973.

[11] D. Ling, Geodesics on surfaces of revolution, Trans. Amer. Math. Soc. 59 (1946) 415-429.

[12] H. von Mangoldt, Über diejenigen Punkte auf positive gekrümmten Flächen, welche die Eigenschaft haben, dass die von Ihnen ausgehenden geodätischen Linien nie aufhören, kürzeste Linien zu sein, J. Reine Angew. Math. 91 (1881) 23-53.

[13] S. B. Meyers, Connection between differential geometry and topology. I, Duke Math. J. 1 (1935) 376-391.

[14] _ Connections between differential geometry and topology. II, Duke Math. J. 2 (1936) 95-102.

[15] H. Poincaré, Sur les lignes géodesiques des surfaces convexes, Trans. Amer. Math. Soc. 6 (1905) 237-274.

[16] W. A. Poor, Some results on nonnegatively curved manifolds, J. Differential Geometry 9 (1974) 583-600.

[17] V. A. Sarafutdinov, Complete open manifolds of nonnegative curvature, Siberian Math. J. 15 (1974) 126-136.

[18] V. A. Toponogov, Riemann spaces with the curvature bounded below, Uspehi Mat. Nauk 14 (1959) 87-130, in Russian.

Hughes Aircraft Company, Culver City, CA 